\title{
Profile expansion for the first nontrivial Steklov eigenvalue in Riemannian manifolds
}

\author{
Mouhamed Moustapha Fall and Tobias Weth
}

\begin{abstract}
We study the problem of maximizing the first nontrivial Steklov eigenvalue of the LaplaceBeltrami Operator among subdomains of fixed volume of a Riemannian manifold. More precisely, we study the expansion of the corresponding profile of this isoperimetric (or isochoric) problem as the volume tends to zero. The main difficulty encountered in our study is the lack of existence results for maximizing domains and the possible degeneracy of the first nontrivial Steklov eigenvalue, which makes it difficult to tackle the problem with domain variation techniques. As a corollary of our results, we deduce local comparison principles for the profile in terms of the scalar curvature on $\mathcal{M}$. In the case where the underlying manifold is a closed surface, we obtain a global expansion and thus a global comparison principle.
\end{abstract}

MSC 2000: 15A42 - 35B05 - 35R45 - 52A40.

\section{Introduction}

Let $(\mathcal{M}, g)$ be a complete Riemannian manifold of dimension $N \geq 2$, and let $\Delta_{g} f=\operatorname{div}_{g}(\nabla f)$ denote the Laplace-Beltrami operator on $\mathcal{M}$. For a bounded regular domain $\Omega \subset \mathcal{M}$ with outer unit normal $\eta$ on $\partial \Omega$, we consider the Steklov eigenvalue problem

$$
\Delta_{g} f=0 \quad \text { in } \Omega, \quad\langle\nabla f, \eta\rangle_{g}=\nu f \quad \text { on } \partial \Omega .
$$

The corresponding set of eigenvalues, counted with multiplicities, is given as an increasing sequence

$$
0=\nu_{1}(\Omega, g)<\nu_{2}(\Omega, g) \leq \cdots+\infty .
$$

In the case where $\mathcal{M}=\mathbb{R}^{N}$, endowed with the euclidean metric $g_{\text {eucl }}$, it has been proved by Brock 2 that, among domains $\Omega$ of fixed volume $v>0$, balls with volume $v$ are the unique maximizers of $\nu_{2}(\Omega)=\nu_{2}\left(\Omega, g_{\text {eucl }}\right)$. In the planar case within the class of simply connected subdomains of $\mathbb{R}^{2}$, this result had been derived earlier by Weinstock 14. The result also extends to the class of simply connected subdomains of a complete Riemannian surface with constant scalar curvature, see [7, Theorem 7]. We point out that, in the euclidean case, Brock [2] actually proved the stronger inequality

$$
\sum_{i=2}^{N+1} \frac{1}{\nu_{i}(\Omega)} \geq N \quad \text { for every domain } \Omega \text { having the same volume as the unit ball } B \subset \mathbb{R}^{N},
$$

with equality if and only if $\Omega=B$. Note that $\nu_{2}(B)=\mu_{3}(B)=\cdots=\mu_{N+1}(B)=1$, and the corresponding eigenfunctions on the unit ball are simply the coordinate functions $x \mapsto x^{i}$, $i=1, \ldots, N$. Xia and Wang (see [15, Theorem 2.1]) also proved a related lower bound for

mouhamed.m.fall@aims-senegal.org (M. M. Fall), weth@math.uni-frankfurt (T. Weth).

African Institute for Mathematical Sciences of Senegal, KM 2, Route de Joal, B. P. 14 18. Mbour, Sénégal.

Goethe-Universität Frankfurt, Institut für Mathematik. Robert-Mayer-Str. 10 D-60054 Frankfurt, Germany. 
$\sum_{i=2}^{N+1} \frac{1}{\nu_{i}(\Omega, g)}$ in the case where $(\mathcal{M}, g)$ is a Hadamard manifold.

In the present paper we study the geometric variational problem of maximizing $\nu_{2}(\Omega, g)$ among domains with fixed small volume in a general Riemannian manifold $(\mathcal{M}, g)$. For $0<v<|\mathcal{M}|_{g}$, we define the Weinstock-Brock profile of $\mathcal{M}$ as

$$
W B_{\mathcal{M}}(v, g):=\sup _{\Omega \subset \mathcal{M},|\Omega|_{g}=v} \nu_{2}(\Omega, g) .
$$

Here and in the following, we assume without further mention that only regular bounded domains $\Omega \subset \mathcal{M}$ are considered, and we let $|\Omega|_{g}$ denote the $N$-dimensional volume with respect to the metric $g$. For open subsets $\mathcal{A} \subset \mathcal{M}$ and $0<v<|\mathcal{A}|_{g}$, we also define

$$
W B_{\mathcal{A}}(v, g):=\sup _{\Omega \subset \mathcal{A},|\Omega|_{g}=v} \nu_{2}(\Omega, g)
$$

assuming again without further mention that only regular bounded domains $\Omega \subset \mathcal{A}$ are considered. By Brock's result 2 mentioned above and the scaling properties of $\nu_{2}$, we then have

$$
W B_{\mathbb{R}^{N}}(v)=\left(\frac{v}{|B|}\right)^{-\frac{1}{N}} .
$$

In our first result we analyze the local effect of the scalar curvature of $\mathcal{M}$ on the $\nu_{2}$-profile. For this we let $B_{g}\left(y_{0}, r\right)$ denote the geodesic ball in $\mathcal{M}$ centered at a point $y_{0} \in \mathcal{M}$ with radius $r$. The following result contains a global asymptotic lower bound for $W B_{\mathcal{M}}(v)$ and a sharp two-sided bound for $W B_{B_{g}\left(y_{0}, r\right)}(v)$ if $r>0$ is small.

Theorem 1.1 Let $\mathcal{M}$ be a complete $N$-dimensional Riemannian manifold with $N \geq 2$, and let $S$ denote the scalar curvature function on $\mathcal{M}$. Moreover, let $y_{0} \in \mathcal{M}$. Then we have:

(i) As $v \rightarrow 0$,

$$
W B_{\mathcal{M}}(v) \geq\left(\frac{v}{|B|}\right)^{-\frac{1}{N}}+\frac{S\left(y_{0}\right)}{2 N(N+2)}\left(\frac{v}{|B|}\right)^{\frac{1}{N}}+o\left(v^{\frac{1}{N}}\right) .
$$

(ii) For every $y_{0} \in \mathcal{M}$ and every $\varepsilon>0$, there exists $r_{\varepsilon}>0$ such that

$$
W B_{B_{g}\left(y_{0}, r_{\varepsilon}\right)}(v)\left\{\begin{array}{l}
\geq\left(\frac{v}{|B|}\right)^{-\frac{1}{N}}+\left(\frac{S\left(y_{0}\right)}{2 N(N+2)}-\varepsilon\right)\left(\frac{v}{|B|}\right)^{\frac{1}{N}} \\
\leq\left(\frac{v}{|B|}\right)^{-\frac{1}{N}}+\left(\frac{S\left(y_{0}\right)}{2 N(N+2)}+\varepsilon\right)\left(\frac{v}{|B|}\right)^{\frac{1}{N}}
\end{array} \text { for } v \in\left(0,\left|B_{g}\left(y_{0}, r_{\varepsilon}\right)\right|_{g}\right) .\right.
$$

We note that $S\left(y_{0}\right)$ in (3) can be replaced by $\sup _{\mathcal{M}} S$ if the supremum is attained on $\mathcal{M}$ (e.g. if $\mathcal{M}$ is compact). The result naturally leads to the question whether a sharp upper bound can also be obtained for $W B_{\mathcal{M}}(v)$. The main problem which arises here is the fact that almost maximizing domains of small volume $v$ do not necessarily have small diameter if $N \geq 3$. However, we are able to control the diameter in the two-dimensional case, and thus we have the following result.

Theorem 1.2 Let $(\mathcal{M}, g)$ be a closed Riemannian surface. Then we have

$$
W B_{\mathcal{M}}(v)=\left(\frac{v}{\pi}\right)^{-\frac{1}{2}}+\frac{S_{\mathcal{M}}}{16}\left(\frac{v}{\pi}\right)^{\frac{1}{2}}+o\left(v^{\frac{1}{2}}\right) \quad \text { as } v \rightarrow 0,
$$

where $S_{\mathcal{M}}$ denotes the maximum of the scalar curvature function $S$ on $\mathcal{M}$.

We conjecture that a similar global expansion holds in closed Riemannian manifolds of higher dimension, but for now this remains open. As we will explain below in more detail, our proof of Theorem 1.2 does not extend to higher dimensions.

An immediate consequence of the asymptotic estimates given in Theorem 1.1 and 1.2 are the following comparison principles. 
Corollary 1.3 Let $\left(\mathcal{M}_{1}, g_{1}\right),\left(\mathcal{M}_{2}, g_{2}\right)$ be two $N$-dimensional complete Riemannian manifolds, $N \geq 2$ with scalar curvature functions $S_{1}, S_{2}$ respectively.

(i) Let $y_{1} \in \mathcal{M}_{1}$ and $y_{2} \in \mathcal{M}_{2}$ such that $S_{1}\left(y_{1}\right)<S_{2}\left(y_{2}\right)$. Then there exists $r>0$ such that

$$
W B_{B_{g_{1}}\left(y_{1}, r\right)}(v)<W B_{B_{g_{2}}\left(y_{2}, r\right)}(v) \quad \text { for any } v \in\left(0, \min \left\{\left|B_{g_{1}}\left(y_{1}, r\right)\right|_{g_{1}},\left|B_{g_{2}}\left(y_{2}, r\right)\right|_{g_{2}}\right\}\right) \text {. }
$$

(ii) If $N=2$ and $\left(\mathcal{M}_{1}, g_{1}\right),\left(\mathcal{M}_{2}, g_{2}\right)$ are closed Riemannian surfaces with $\max _{M_{1}} S_{1}<\max _{M_{2}} S_{2}$, then there exists $r>0$ such that

$$
W B_{\mathcal{M}_{1}}(v)<W B_{\mathcal{M}_{2}}(v) \quad \text { for any } 0<v<r .
$$

Our results should be seen in comparison with our recent work [8] on the Szegö-Weinberger profile in Riemannian manifolds, which arises from the corresponding maximization problem for the first nontrivial Neumann eigenvalue of $-\Delta_{g}$ on $\mathcal{M}$. In this work we established an analogue of Theorem 1.1 for the the Szegö-Weinberger profile. Similarly as in [8], the first step in the proof of Theorem 1.1 is the derivation of expansions for $\nu_{2}$ for small geodesic balls and small ellipsoids with small eccentricity centered at a point $y_{0} \in \mathcal{M}$. More precisely, in Proposition 2.2 below we show that

$$
\nu_{2}\left(B_{g}\left(y_{0}, r\right), g\right)=\frac{1}{r}+\frac{2 r}{3(N+2)} R_{\min }\left(y_{0}\right)+o(r) \quad \text { as } r \rightarrow 0
$$

with $R_{\min }\left(y_{0}\right):=\min _{A \in T_{y_{0}} \mathcal{M},|A|_{g}=1} \operatorname{Ric}_{y_{0}}(A, A)$. Hence there is an anisotropic curvature effect on the expansion which suggests that small geodesic balls are not optimal up to linear order in $r$ for the maximization problem. We therefore construct a family (depending on $r$ ) of small ellipsoids $E\left(y_{0}, r\right)$ which are choosen such that the eccentricity balances the anisotropic curvature effects, so that the resulting expansion

$$
\nu_{2}\left(E\left(y_{0}, r\right), g\right)=\frac{1}{r}+\frac{2 r}{3 N(N+2)} S\left(y_{0}\right)+o(r)
$$

depends only on the scalar curvature $S\left(y_{0}\right)$, see Proposition 2.5 below. The computations of these expansions bear some similarities with the corresponding ones in [8, although some differences arise due to the fact that boundary integrals have to be expanded in the present case. On the other hand, we note that the simple form of the eigenfunctions corresponding to $\nu_{2}(B)$ leads to a nicer expansion than in the Neumann eigenvalue case. We shall see that by combining (6) with the volume expansion for $E\left(y_{0}, r\right)$, we already obtain the lower bound for the profile given in Theorem 1.1(i). The proof of the local upper bound in Theorem 1.1(ii) is more involved and proceeds eventually by a contradiction argument. For this, some care is needed to construct, for given subdomains of $B_{g}\left(y_{0}, r\right)$ with $r>0$ small, suitable vector fields which can be used in combination with the variational principle for $\nu_{2}$ in order to control the symmetric distance of these domains to a suitably chosen geodesic ball with the same volume. Within this step, the key tool is a quantitative weighted isoperimetric inequality proved recently by Brasco, de Philipps and Ruffini see [1, Theorem B].

We point out that, in the proof of the local upper bound for the profile given in Theorem 1.1(ii), the arguments differ significantly from the ones in [8] for the Neumann eigenvalue case. We also remark that, at least up to now, Theorem 1.2 has no analogue for the corresponding Neumann eigenvalue profile. The proof of this global expansion is technically involved, but the strategy is easy to explain. We will show that almost maximizing domains for $\nu_{2}$ of small (fixed) volume must also have small diameter. There is no hope to prove this in dimension $N \geq 3$, since in this case one may increase the diameter of the domain by adding a long cusp of small volume and perimeter. By the variational characterization, this will only result in a small change of $\nu_{2}$. In contrast, as remarked before, in the two-dimensional case we will be able to deduce bounds on the diameter with the help of the variational characterization of $\nu_{2}$ and suitably constructed test functions.

To close the introduction, we mention the earlier work in [5, 9] on the small volume expansion for the Faber-Krahn profile, which is related to the minimization of the first Dirichlet eigenvalue 
$\lambda_{1}(\Omega, g)$ of $-\Delta_{g}$ among subdomains $\Omega$ of fixed volume. One important difference between $\lambda_{1}(\Omega, g)$ and $\nu_{2}(\Omega, g)$ is the degeneracy of $\nu_{2}$ in the case of the unit ball and possibly also in the case of maximizing domains on Riemannian manifolds. This degeneracy makes it difficult to apply domain variation arguments to the maximization problem.

The paper is organized as follows. Section 2 contains some preliminaries and the proof of local expansions of $\nu_{2}$ for small geodesic balls and small ellipsoids with small eccentricity. In particular, as already remarked above, we shall see that suitably chosen ellipsoids provide the optimal lower bound in Theorem 1.1(i). In Section 3 we then complete the proof of Theorem 1.1 by providing the upper bound in (ii). Finally, in Section 4 , we focus on the two-dimensional case $N=2$ and give the proof Theorem 1.2

Acknowledgments: The authors would like to thank Lorenzo Brasco for many helpful comments on previous versions of the paper, stimulating discussions, and for drawing our attention to the paper [1]. The first author is supported by the Alexander von Humboldt foundation. Part of the paper was written while the second author was visiting AIMS Senegal in November 2014 within the project "Joint steps in geometric variational problems" funded by DAAD, Germany. He wishes to thank AIMS Senegal for the kind hospitality and DAAD for the funding of the visit.

General Notation: Throughout the paper, we let $B$ denote the open unit ball in $\mathbb{R}^{N}$ and put $r B:=\left\{x \in \mathbb{R}^{N}:|x|<r\right\}$ for $r>0$. Moreover, we write $x \cdot y$ for the euclidean scalar product of $x, y \in \mathbb{R}^{N}$.

\section{Local expansions of $\nu_{2}$ for small geodesic balls and ellip- soids.}

Let $(\mathcal{M}, g)$ be a complete Riemannian manifold of dimension $N \geq 2$. For a smooth bounded subdomain domain $\Omega$ of $(\mathcal{M}, g)$, we write $\nu_{2}=\nu_{2}(\Omega, g)$ for the first nontrivial eigenvalue of (1). The variational characterization of $\nu_{2}(\Omega, g)$ is given by

$$
\nu_{2}(\Omega, g)=\inf \left\{\int_{\Omega}|\nabla u|_{g}^{2} d v_{g}: u \in H^{1}(\Omega), \int_{\partial \Omega} u^{2} d \sigma_{g}=1, \int_{\partial \Omega} u d \sigma_{g}=0\right\} .
$$

Here $v_{g}$ denotes the volume element of the metric $g$, and $\sigma_{g}$ denotes the volume element of the restriction of $g$ to an $N-1$-dimensional submanifold of $\mathcal{M}$. For a Borel subset $A \subset \mathcal{M}$, we let $|A|_{g}$ denote the $N$-dimensional volume of $\Omega$ and $\sigma_{g}(A)$ denote the $N$-1-dimensional Haussdorffmeasure, both with respect to the metric $g$. If $\mathcal{M}=\mathbb{R}^{N}$ and $g$ is the Euclidean metric, we simply write $d x$ in place of $d v_{g},|\cdot|$ in place of $|\cdot|_{g}, d \sigma$ in place of $d \sigma_{g}$ and $\nu_{2}(\Omega)$ in place of $\nu_{2}(\Omega, g)$. We recall that the minimizers of the minimization problem (17) are precisely the eigenfunctions corresponding to $\nu_{2}(\Omega, g)$. As noted already, in the case of the unit ball $B \subset \mathbb{R}^{N}$ we have that $\nu_{2}(B)=1$ is of multiplicity $N$ with corresponding eigenfunctions given by $x \mapsto x^{i}, i=1, \ldots, N$.

In the following, we assume that $(\mathcal{M}, g)$ is complete, and we fix $y_{0} \in \mathcal{M}$ and an orthonormal basis $E_{1}, \ldots, E_{N}$ of $T_{y_{0}} \mathcal{M}$. We will use the (somewhat sloppy) notation

$$
X:=x^{i} E_{i} \in T_{y_{0}} \mathcal{M} \quad \text { for } x \in \mathbb{R}^{N} .
$$

Here and in the following, we sum over repeated upper and lower indices as usual. We consider the map

$$
\Psi: \mathbb{R}^{N} \rightarrow \mathcal{M}, \quad \Psi(x):=\operatorname{Exp}_{y_{0}}(X),
$$

which gives rise to a local geodesic coordinate system of a neighborhood of $y_{0}$. A geodesic ball in $\mathcal{M}$ centered at $y_{0}$ with radius $r>0$ is given as $B_{g}\left(y_{0}, r\right)=\Psi(r B)$. The map $\Psi$ induces coordinate vector fields $Y_{i}:=\Psi_{*} \frac{\partial}{\partial x^{i}}$ on $\mathbb{R}^{N}$ given by

$$
Y_{i}(x)=d \operatorname{Exp}_{y_{0}}(X) E_{i} \in T_{\Psi(x)} \mathcal{M}, \quad \text { for } x \in \mathbb{R}^{N}, i=1, \ldots, N .
$$


We need local expansions for the associated metric coefficients

$$
g_{i j}(x)=\left\langle Y_{i}(x), Y_{j}(x)\right\rangle_{g} \quad \text { for } x \in \mathbb{R}^{N}, i . j=1, \ldots, N .
$$

For this we let $R_{y_{0}}: T_{y_{0}} \mathcal{M} \times T_{y_{0}} \mathcal{M} \times T_{y_{0}} \mathcal{M} \rightarrow T_{y_{0}} \mathcal{M}$ denote the Riemannian curvature tensor at $y_{0}$ and

$$
R i c_{y_{0}}: T_{y_{0}} \mathcal{M} \times T_{y_{0}} \mathcal{M} \rightarrow \mathbb{R}, \quad \operatorname{Ric}_{y_{0}}(X, Y)=-\sum_{i=1}^{N}\left\langle R_{y_{0}}\left(X, E_{i}\right) Y, E_{i}\right\rangle_{g}
$$

the Ricci tensor at $y_{0}$. Moreover, we let $S: \mathcal{M} \rightarrow \mathbb{R}$ denote the scalar curvature function on $\mathcal{M}$, so that $S\left(y_{0}\right)=\sum_{k=1}^{N} R i c_{y_{0}}\left(E_{k}, E_{k}\right)$. It will be useful to put

$$
R_{i j k l}:=\left\langle R_{y_{0}}\left(E_{i}, E_{j}\right) E_{k}, E_{l}\right\rangle_{g} \quad \text { and } \quad R_{i j}:=\operatorname{Ric}_{y_{0}}\left(E_{i}, E_{j}\right) \quad \text { for } i, j=1, \ldots, N \text {. }
$$

Without changing the value of these constants, we sometimes raise lower to upper indices in the following. We then have the following well known local expansions as $|x| \rightarrow 0$ (see e.g. in [4, §II.8]):

$$
\begin{aligned}
& g_{i j}(x)=\delta_{i j}+\frac{1}{3}\left\langle R_{y_{0}}\left(X, E_{i}\right) X, E_{j}\right\rangle_{g}+O\left(|x|^{3}\right)=\delta_{i j}+\frac{1}{3} R_{k i l j} x^{k} x^{l}+O\left(|x|^{3}\right) \\
& d v_{g}(x)=\left(1-\frac{1}{6} \operatorname{Ric}_{y_{0}}(X, X)+O\left(|x|^{3}\right)\right) d x=\left(1-\frac{1}{6} R_{l k} x^{l} x^{k}+O\left(|x|^{3}\right)\right) d x .
\end{aligned}
$$

As a consequence of (11), the volume expansion of metric balls is given by

$$
\left|B_{g}\left(y_{0}, r\right)\right|_{g}=r^{N}|B|\left(1-\frac{1}{6(N+2)} r^{2} S\left(y_{0}\right)+O\left(r^{4}\right)\right) .
$$

The first goal of this section is to derive an expansion for $\nu_{2}$ on small geodesic balls centered at $y_{0}$. It will be useful to pull back the problem to the unit ball $B \subset \mathbb{R}^{N}$. For this we let $r>0$ be smaller than half of the injectivity radius of $\mathcal{M}$ at $y_{0}$, so that $B_{g}\left(y_{0}, s\right)$ is a regular domain for $s \leq 2 r$. Moreover, we consider the pull back metric of $g$ under the map $2 B \rightarrow \mathcal{M}, x \mapsto \Psi(r x)$, rescaled with the factor $\frac{1}{r^{2}}$. Denoting this metric on $2 B$ by $g_{r}$, we then have, in euclidean coordinates,

$$
\left[g_{r}\right]_{i j}(x)=\left.\left\langle\frac{\partial}{\partial x^{i}}, \frac{\partial}{\partial x^{j}}\right\rangle_{g_{r}}\right|_{x}=\left\langle Y_{i}(\Psi(r x)), Y_{j}(\Psi(r x))\right\rangle_{g}=g_{i j}(r x),
$$

so that, as a consequence of (10),

$$
\left[g_{r}\right]_{i j}(x)=\delta_{i j}+\frac{r^{2}}{3} R_{k i l j} x^{k} x^{l}+O\left(r^{3}\right) \quad \text { as } r \rightarrow 0
$$

and

$$
g_{r}^{i j}(x)=\delta^{i j}-\frac{r^{2}}{3} R_{k l}^{i j} x^{k} x^{l}+O\left(r^{3}\right) \quad \text { as } r \rightarrow 0
$$

uniformly for $x \in \bar{B}$. Here, as usual, $\left(g_{r}^{i j}\right)_{i j}$ denotes the inverse of the matrix $\left(\left[g_{r}\right]_{i j}\right)_{i j}$. Setting $\left|g_{r}\right|=\operatorname{det}\left(\left[g_{r}\right]_{i j}\right)_{i j}$, we also have

$$
\sqrt{\left|g_{r}\right|}(x)=1-\frac{r^{2}}{6} R_{k l} x^{k} x^{l}+O\left(r^{3}\right) \quad \text { as } r \rightarrow 0
$$

uniformly for $x \in \bar{B}$ by (11). Since this expansion is valid in the sense of $C^{1}$-functions on $\bar{B}$, it follows that

$$
\frac{\partial}{\partial x^{i}} \sqrt{\left|g_{r}\right|}=-\frac{r^{2}}{3} R_{k i} x^{k}+O\left(r^{3}\right) \quad \text { as } r \rightarrow 0 \text { for } i=1, \ldots, N .
$$

The expansion (15) obviously yields

$$
d v_{g_{r}}(x)=\left(1-\frac{r^{2}}{6} R_{l k} x^{l} x^{k}+O\left(r^{3}\right)\right) d x \quad \text { as } r \rightarrow 0
$$

uniformly for $x \in \bar{B}$. We will also need the following expansion for boundary integrals with respect to subdomains of $B$. 
Lemma 2.1 For every smooth domain $U \subset B$ and every $f \in C^{1}(\partial U)$ we have

$$
\int_{\partial U} f(x) d \sigma_{g_{r}}=\left(1+O\left(r^{2}\right)\right) \int_{\partial U} f(x) d \sigma
$$

where $\frac{O\left(r^{2}\right)}{r^{2}}$ remains bounded uniformly in $U$ and $f$ as $r \rightarrow 0$. Moreover, for every $f \in C^{1}(\partial B)$ we have

$$
\int_{\partial B} f(x) d \sigma_{g_{r}}(x)=\int_{\partial B}\left(1-\frac{r^{2}}{6} R_{l k} x^{l} x^{k}\right) f(x) d \sigma+O\left(r^{3}\right) \int_{\partial B} f(x) d \sigma
$$

where $\frac{O\left(r^{3}\right)}{r^{3}}$ remains bounded uniformly in $f$ as $r \rightarrow 0$.

We note that (19) follows from the computations in [12, Appendix 4.1]. Here we provide a different short proof, based on integration by parts.

Proof. Let $\eta_{r}$ denote the unit outer normal vector field on $\partial U$ with respect to $g_{r}$ and $\eta$ the unit outer normal vector field on $\partial U$ with respect to the euclidean metric. We first claim that, for fixed $r>0$,

$$
\int_{\partial U} f d \sigma_{g_{r}}=\int_{\partial U_{r}} f \sqrt{\left|g_{r}\right|} \eta_{r} \cdot \eta d \sigma \quad \text { for every } f \in C^{1}(\partial U)
$$

where, as before, - denotes the euclidean scalar product. To show this, we may first extend $f \eta_{r}: \partial U \rightarrow \mathbb{R}^{N}$ to a $C^{1}$-vector field $\xi$ on $\mathbb{R}^{N}$. Applying the divergence theorem with respect to the metric $g_{r}$, we then have

$$
\int_{U} \operatorname{div}_{g_{r}} \xi \sqrt{\left|g_{r}\right|} d x=\int_{U} \operatorname{div}_{g_{r}} \xi d v_{g_{r}}=\int_{\partial U}\left\langle\xi, \eta_{r}\right\rangle_{g_{r}} d \sigma_{g_{r}}=\int_{\partial U} f d \sigma_{g_{r}} .
$$

On the other hand, applying the divergence theorem with respect to the euclidean metric, we find that

$$
\int_{U} \operatorname{div}_{g_{r}} \xi \sqrt{\left|g_{r}\right|} d x=\int_{U} \frac{\partial}{\partial x^{i}}\left[\xi^{i} \sqrt{\left|g_{r_{k}}\right|}\right] d x=\int_{\partial U} \sqrt{\left|g_{r_{k}}\right|} \xi \cdot \eta d \sigma=\int_{\partial U} f \sqrt{\left|g_{r_{k}}\right|} \eta_{r} \cdot \eta d \sigma .
$$

Hence (20) follows. In order to expand the term $\eta_{r} \cdot \eta$ in $r$, we consider a point $q \in \partial U$ and let $e_{i}, i=1, \ldots, N-1$ be an orthonormal basis of $T_{q} \partial U$ with respect to the Euclidean metric. For simplicity, we will write $\eta_{r}$ and $\eta$ instead of $\eta_{r}(q)$ and $\eta(q)$ in the following. We then have that

$$
\eta_{r}=\left[\eta_{r} \cdot \eta\right] \eta+\left[\eta_{r} \cdot e_{i}\right] e_{i}
$$

Since $e_{i} \in T_{q} \partial U$, we have, by (13),

$$
0=\left\langle\eta_{r}, e_{i}\right\rangle_{g_{r}}=\eta_{r} \cdot e_{i}+O\left(r^{2}\right)\left|\eta_{r}\right|\left|e_{i}\right|=\eta_{r} \cdot e_{i}+O\left(r^{2}\right)\left|\eta_{r}\right|
$$

Moreover, (13) also implies that

$$
1=\left|\eta_{r}\right|_{g_{r}}^{2}=\left|\eta_{r}\right|^{2}+\frac{r^{2}}{3} R_{k i l j} \eta_{r}^{i} \eta_{r}^{j} q^{k} q^{l}+O\left(r^{3}\right)\left|\eta_{r}\right|^{2}=\left(1+O\left(r^{2}\right)\right)\left|\eta_{r}\right|^{2}
$$

and hence

$$
\left|\eta_{r}\right|=1+O\left(r^{2}\right)
$$

Consequently, (23) implies that $\eta_{r} \cdot e_{i}=O\left(r^{2}\right)$ independently of $U \subset B, q$ and the choice of the orthonormal basis $e_{i}$. Taking the euclidean scalar product of (22) with $\eta_{r}$, we now find that

$$
\left|\eta_{r}\right|^{2}=\left[\eta_{r} \cdot \eta\right]^{2}+O\left(r^{4}\right)
$$

Together with (25) this implies that $\left[\eta_{r} \cdot \eta\right]^{2}=1+O\left(r^{2}\right)$. Since both $\eta_{r}$ and $\eta$ are defined as outer normal vector fields, we conclude that

$$
\eta_{r} \cdot \eta=1+O\left(r^{2}\right) \quad \text { uniformly on } \partial U \text { and independently of } U \text {. }
$$


Combining this with (15) and (20), we obtain (18).

To see (19), we consider the special case $U=B$, and we note that, as a consequence of Gauss' Lemma (see e.g. [11, Corollary 5.2.3]), we have that $\eta_{r}(q)=q=\eta(q)$ for every $q \in \partial B$. Together with (15) and (20) this implies (19).

We are now ready to establish the desired expansion for $\nu_{2}$ on small geodesic balls.

Proposition 2.2 We have

$$
\nu_{2}\left(B_{g}\left(y_{0}, r\right), g\right)=\frac{1}{r}+\frac{2 r}{3(N+2)} R_{\min }\left(y_{0}\right)+o(r) \quad \text { as } r \rightarrow 0
$$

with $R_{\text {min }}\left(y_{0}\right):=\min _{A \in T_{y_{0}} \mathcal{M},|A|_{g}=1} \operatorname{Ric}_{y_{0}}(A, A)$.

Proof. Let $u_{r} \in C^{3}\left(\overline{B_{g}\left(y_{0}, r\right)}\right)$ be an eigenfunction associated to $\nu_{2}\left(B_{g}\left(y_{0}, r\right), g\right)$, i.e., we have

$$
\Delta_{g} u_{r}=0 \quad \text { in } B_{g}\left(y_{0}, r\right), \quad\left\langle\nabla u_{r}, \eta_{r}\right\rangle_{g}=\nu_{2}\left(B_{g}\left(y_{0}, r\right), g\right) u_{r} \quad \text { on } \partial B_{g}\left(y_{0}, r\right),
$$

where $\eta_{r}$ denotes the outer unit normal on $\partial B_{g}\left(y_{0}, r\right)$. Then the function $\Phi_{r}: \bar{B} \rightarrow \mathbb{R}, \Phi_{r}(x)=$ $r^{\frac{N}{2}} u_{r}(\Psi(r x))$ satisfies

$$
\Delta_{g_{r}} \Phi_{r}=0 \quad \text { in } B, \quad\left\langle\nabla \Phi_{r}, \eta\right\rangle_{g_{r}}=\nu_{2}\left(B, g_{r}\right) \Phi_{r} \quad \text { on } \partial B,
$$

with

$$
\Delta_{g_{r}} \Phi_{r}=\frac{1}{\sqrt{\left|g_{r}\right|}} \frac{\partial}{\partial x^{i}}\left(\sqrt{\left|g_{r}\right|} g_{r}^{i j} \frac{\partial \Phi_{r}}{\partial x^{j}}\right) \quad \text { and } \quad \nu_{2}\left(B, g_{r}\right)=r \nu_{2}\left(B_{g}\left(y_{0}, r\right), g\right) .
$$

Hence the asserted expansion (28) is equivalent to

$$
\nu_{2}\left(B, g_{r}\right)=1+\frac{2 r^{2}}{3(N+2)} R_{\min }\left(y_{0}\right)+o\left(r^{2}\right) .
$$

Moreover, by normalization we may assume that $\int_{\partial B} \Phi_{r}^{2} d \sigma_{g_{r}}=1$. To prove (30), we first note that, since $g_{r}$ converges to the Euclidean metric in $\bar{B}$, it follows from the variational characterization of $\nu_{2}$ that $\nu_{2}\left(B, g_{r}\right) \rightarrow \nu_{2}(B)=1$. Moreover, by using standard elliptic regularity theory, one may show that, along a sequence $r_{k} \rightarrow 0$, we have $\Phi_{r_{k}} \rightarrow \Phi$ in $H^{1}(B)$ for some function $\Phi \in C_{l o c}^{2}(B) \cap C^{1}(\bar{B})$ satisfying

$$
\Delta \Phi=0 \quad \text { in } B, \quad\langle\nabla \Phi, \eta\rangle=\Phi \quad \text { on } \partial B, \quad \text { and } \quad \int_{\partial B} \Phi^{2} d \sigma=1 .
$$

Hence there exists $a=\left(a_{1}, \ldots, a_{N}\right)=\left(a^{1}, \ldots, a^{N}\right) \in \mathbb{R}^{N}$ with $|a|=1$ and such that

$$
\Phi(x)=\frac{a \cdot x}{\sqrt{|B|}} \quad \text { for } x \in \bar{B} .
$$

For matters of convenience, we will continue to write $r$ instead of $r_{k}$ in the following. By integration by parts in (29), using $\left\langle\nabla \Phi_{r}, \eta\right\rangle_{g_{r}}=0$ and $d v_{g_{r}}=\sqrt{\left|g_{r}\right|} d x$, we have

$$
\nu_{2}\left(B, g_{r}\right) \int_{\partial B} \Phi \Phi_{r} d \sigma_{g_{r}}=\int_{B} \sqrt{\left|g_{r}\right|} g_{r}^{i j} \frac{\partial \Phi}{\partial x^{i}} \frac{\partial \Phi_{r}}{\partial x^{j}} d x .
$$

We thus find, using (14), (16), (31) and integrating by parts again,

$$
\begin{aligned}
& \int_{B} \sqrt{\left|g_{r}\right|} g_{r}^{i j} \frac{\partial \Phi}{\partial x^{i}} \frac{\partial \Phi_{r}}{\partial x^{j}} d x=\int_{B} \sqrt{\left|g_{r}\right|}\left(\nabla \Phi_{r} \cdot \nabla \Phi-\frac{r^{2}}{3} R_{k l}^{i j} x^{k} x^{l} \frac{\partial \Phi}{\partial x^{i}} \frac{\partial \Phi_{r}}{\partial x^{j}}\right) d x+O\left(r^{3}\right) \\
& =\int_{\partial B} \Phi \Phi_{r} d \sigma_{g_{r}}-\int_{B} \Phi_{r} \nabla \sqrt{\left|g_{r}\right|} \cdot \nabla \Phi d x-\frac{r^{2}}{3} \int_{B}\left\langle R_{k l}^{i j} x^{k} x^{l} \frac{\partial \Phi}{\partial x^{i}} \frac{\partial \Phi_{r}}{\partial x^{j}} d x+O\left(r^{3}\right)\right. \\
& =\int_{\partial B} \Phi \Phi_{r} d \sigma_{g_{r}}+\frac{r^{2}}{3} \int_{B} \Phi_{r} R_{i}{ }^{j} x^{i} \frac{\partial \Phi}{\partial x^{j}} d x-\frac{r^{2}}{3} \int_{B} R_{k l}^{i j} x^{k} x^{l} \frac{\partial \Phi}{\partial x^{i}} \frac{\partial \Phi_{r}}{\partial x^{j}} d x+O\left(r^{3}\right) .
\end{aligned}
$$


Therefore, since $\int_{\partial B} \Phi \Phi_{r} d \sigma_{g_{r}} \rightarrow 1$ and $\Phi_{r} \rightarrow \Phi$ in $H^{1}(B)$ as $r \rightarrow 0$, we obtain

$$
\begin{aligned}
\nu_{2}\left(B, g_{r}\right) & =1+\frac{r^{2}}{3} \int_{B} \Phi R_{i}{ }^{j} x^{i} \frac{\partial \Phi}{\partial x^{j}} d x-\frac{r^{2}}{3} \int_{B} R_{k l}{ }^{i j} x^{k} x^{l} \frac{\partial \Phi}{\partial x^{i}} \frac{\partial \Phi}{\partial x^{j}} d x+o\left(r^{2}\right) \\
& =1+\frac{r^{2}}{3|B|} \int_{B} a_{k} x^{k} R_{i}{ }^{j} x^{i} a_{j} d x-\frac{r^{2}}{3|B|} \int_{B} R_{k l}{ }^{j} x^{k} x^{l} a_{i} a_{j} d x+o\left(r^{2}\right) .
\end{aligned}
$$

Recalling that

$$
\int_{B} x^{i} x^{j} d x=\delta^{i j} \frac{|B|}{N+2} \quad \text { for } i, j=1, \ldots, N
$$

we calculate

$$
\int_{B} a_{k} x^{k} R_{i}{ }^{j} x^{i} a_{j} d x=\frac{|B|}{N+2} R^{k j} a_{k} a_{j}=\frac{|B|}{N+2} \operatorname{Ric}_{y_{0}}(A, A)
$$

with $A:=a_{i} E^{i}$ and

$$
\int_{B} R_{k l}^{i j} x^{k} x^{l} a_{i} a_{j} d x=-\frac{|B|}{N+2} R^{i j} a_{i} a_{j}=-\frac{|B|}{N+2} \operatorname{Ric}_{y_{0}}(A, A) .
$$

Therefore

$$
\nu_{2}\left(B, g_{r}\right)=1+\frac{2 r^{2}}{3(N+2)} \operatorname{Ric}_{y_{0}}(A, A)+o\left(r^{2}\right) .
$$

We now need to recall that - more precisely - here we consider a sequence $r=r_{k} \rightarrow 0$. Nevertheless, the argument implies that

$$
\nu_{2}\left(B, g_{r}\right) \geq 1+\frac{2 r^{2}}{3(N+2)} R_{\min }\left(y_{0}\right)+o\left(r^{2}\right) \quad \text { as } r \rightarrow 0 .
$$

Indeed, if - arguing by contradiction - there is a sequence $r_{k} \rightarrow 0$ such that

$$
\limsup _{k \rightarrow \infty} \nu_{2}\left(B, g_{r}\right)<1+\frac{2 r_{k}^{2}}{3(N+2)} R_{\min }\left(y_{0}\right)+o\left(r_{k}^{2}\right) \quad \text { as } k \rightarrow \infty .
$$

then, by the above argument, there exists a subsequence along which the expansion (38) holds with some $A \in T_{y_{0}} \mathcal{M}$ with $|A|=1$, thus contradicting (40). Hence (39) is true, and it thus remains to prove that

$$
\nu_{2}\left(B, g_{r}\right) \leq 1+\frac{2 r^{2}}{3(N+2)} \operatorname{Ric}_{y_{0}}(A, A)+o\left(r^{2}\right) \quad \text { for all } A \in T_{y_{0}} \mathcal{M} \text { with }|A|=1 .
$$

So consider $a=\left(a^{1}, \ldots, a^{N}\right) \in \mathbb{R}^{N}$ arbitrary with $|a|=1$, let $A=a^{i} E_{i} \in T_{y_{0}} \mathcal{M}$, and define $\Phi: \bar{B} \rightarrow \mathbb{R}$ by (32). Moreover, put $c_{r}:=\frac{1}{|\partial B|_{g_{r}}} \int_{\partial B} \Phi d \sigma_{g_{r}}$ for $r>0$ small. Then, by (19),

$c_{r}=\left(\frac{1}{|\partial B|}+O\left(r^{2}\right)\right)\left(\int_{\partial B} \Phi(x)\left[1-\frac{1}{6} R_{k l} x^{k} x^{l}\right] d \sigma(x)+O\left(r^{3}\right)\right)=\left(\frac{1}{|\partial B|}+O\left(r^{2}\right)\right) O\left(r^{3}\right)=O\left(r^{3}\right)$,

since the function $x \mapsto \Phi(x)\left[1-\frac{1}{6} R_{k l} x^{k} x^{l}\right]$ is odd with respect to reflection at the origin. Hence, using the variational characterization of $\nu_{2}\left(B, g_{r}\right)$, we find that

$$
\nu_{2}\left(B, g_{r}\right) \leq \frac{\int_{B}\left|\nabla\left(\Phi-c_{r}\right)\right|_{g_{r}}^{2} d v_{g_{r}}}{\int_{\partial B}\left(\Phi-c_{r}\right)^{2} d \sigma_{g_{r}}}=\frac{\int_{B}|\nabla \Phi|_{g_{r}}^{2} d v_{g_{r}}}{\int_{\partial B}\left[\Phi^{2}+O\left(r^{3}\right)\right] d \sigma_{g_{r}}}=\frac{\int_{B}|\nabla \Phi|_{g_{r}}^{2} d v_{g_{r}}+O\left(r^{3}\right)}{\int_{\partial B} \Phi^{2} d \sigma_{g_{r}}}
$$

and therefore

$$
\nu_{2}\left(B, g_{r}\right) \int_{\partial B} \Phi^{2} d \sigma_{g_{r}} \leq \int_{B_{g}\left(y_{0}, r\right)}|\nabla \Phi|_{g_{r}}^{2} d v_{g_{r}}+O\left(r^{3}\right)=\int_{B} \sqrt{\left|g_{r}\right|} g_{r}^{i j} \frac{\partial \tilde{\Phi}}{\partial x^{i}} \frac{\partial \Phi}{\partial x^{j}} d x+O\left(r^{3}\right) .
$$

It is by now straightforward that the same estimates as above - starting from (33) - hold with both $\Phi_{r}$ and $\Phi$ replaced by $\Phi$. We thus obtain (41), as required. 
Corollary 2.3 We have

$$
\nu_{2}\left(B_{g}\left(y_{0}, r\right), g\right)=\left(\frac{v}{|B|}\right)^{-\frac{1}{N}}+\frac{4 N R_{\min }\left(y_{0}\right)-S\left(y_{0}\right)}{6 N(N+2)}\left(\frac{v}{|B|}\right)^{\frac{1}{N}}+o\left(v^{\frac{1}{N}}\right)
$$

as $v=\left|B_{g}\left(y_{0}, r\right)\right|_{g} \rightarrow 0$.

ProOF. By the volume expansion (12) of geodesic balls we have

$$
\begin{aligned}
\frac{1}{r}\left(\frac{v}{|B|}\right)^{\frac{1}{N}}=\left(\frac{\left|B_{g}\left(y_{0}, r\right)\right| g}{r^{N}|B|}\right)^{\frac{1}{N}} & =1-\frac{1}{6 N(N+2)} S\left(y_{0}\right) r^{2}+o\left(r^{2}\right) \\
& =1-\frac{1}{6 N(N+2)} S\left(y_{0}\right)\left(\frac{v}{|B|}\right)^{\frac{2}{N}}+o\left(\frac{v}{|B|}\right)^{\frac{2}{N}}
\end{aligned}
$$

as $v=\left|B_{g}\left(y_{0}, r\right)\right|_{g} \rightarrow 0$. Combining this with Proposition 2.2, we get the result.

Next, we wish to derive an expansion of $\nu_{2}$ on small geodesic ellipsoids centered at $y_{0} \in \mathcal{M}$. For this we assume in the following that the orthonormal basis $E_{i}, i=1, \ldots, N$ of $T_{y_{0}} \mathcal{M}$ is chosen such that

$$
R_{i j}=0 \quad \text { for } i \neq j \text {. }
$$

Moreover we put

$$
b_{i}=b^{i}:=\frac{1}{3(N+2)}\left(R_{i i}-\frac{S\left(y_{0}\right)}{N}\right) \quad \text { for } i=1, \ldots, N
$$

and we note that $\sum_{i=1}^{N} b_{i}=0$ since $S\left(y_{0}\right)=\sum_{i=1}^{N} R_{i i}$. For $r>0$ small, we then consider the geodesic ellipsoids $E\left(y_{0}, r\right):=F_{r}(B) \subset \mathcal{M}$, where

$$
F_{r}: B \rightarrow \mathcal{M}, \quad F_{r}(x)=\operatorname{Exp}_{y_{0}}\left(r\left(1+r^{2} b_{i}\right) x^{i} E_{i}\right) .
$$

The special choice of the values $b_{i}$ gives rise to the following asymptotic expansion depending only on the scalar curvature at $y_{0}$.

Proposition 2.4 As $r \rightarrow 0$, we have

$$
\nu_{2}\left(E\left(y_{0}, r\right), g\right)=\frac{1}{r}+\frac{2 r}{3 N(N+2)} S\left(y_{0}\right)+o(r)
$$

and

$$
\left|E\left(y_{0}, r\right)\right|_{g}=\left|B_{g}\left(y_{0}, r\right)\right|_{g}+O\left(r^{N+4}\right)=r^{N}|B|\left(1-\frac{1}{6(N+2)} r^{2} S\left(y_{0}\right)+O\left(r^{4}\right)\right) .
$$

Proof. We consider the pull back metric $h_{r}$ on $B$ of $g$ under the map $F_{r}$ rescaled with the factor $\frac{1}{r^{2}}$. Then we have

$$
\begin{aligned}
{\left[h_{r}\right]_{i j}(x) } & =\left(1+r^{2} b_{i}\right)\left(1+r^{2} b_{j}\right)\left[g_{r}\right]_{i j}\left(\left(1+r^{2} b_{k}\right) x^{k} e_{k}\right)=\left[g_{r}\right]_{i j}(x)+r^{2}\left(b_{i}+b_{j}\right) \delta_{i j}+O\left(r^{4}\right) \\
& =\delta_{i j}+r^{2}\left(\frac{1}{3} R_{k i l j} x^{k} x^{l}+2 b_{i} \delta_{i j}\right)+O\left(r^{3}\right)
\end{aligned}
$$

uniformly in $x \in B$ (where $g_{r}$ is defined as in the proof of Proposition 2.2). Setting $\left|h_{r}\right|=$ $\operatorname{det}\left(\left[h_{r}\right]_{i j}\right)_{i j}$, we deduce the expansion

$$
\left|h_{r}\right|(x)=\left|g_{r}\right|(x)+2 r^{2} \sum_{i=1}^{N} b_{i}+O\left(r^{4}\right)=\left|g_{r}\right|(x)+O\left(r^{4}\right) \quad \text { for } x \in B .
$$

This implies that

$$
\left|E\left(y_{0}, r\right)\right|_{g}=r^{N}|B|_{h_{r}}=r^{N}\left(|B|_{g_{r}}+O\left(r^{4}\right)\right)=\left|B_{g}\left(y_{0}, r\right)\right|_{g}+O\left(r^{N+4}\right),
$$


as claimed in (46). We now turn to (45). For this we first note that, denoting by $\left(h_{r}^{i j}\right)_{i j}$ the inverse of the matrix $\left(\left[h_{r}\right]_{i j}\right)_{i j}$, we have

$$
h_{r}^{i j}(x)=\delta^{i j}-r^{2}\left(\frac{1}{3} R_{k l}^{i j} x^{k} x^{l}+2 b_{i} \delta_{i j}\right)+O\left(r^{3}\right)
$$

by (47), whereas (16) and (48) yield

$$
\frac{\partial}{\partial x^{i}} \sqrt{\left|h_{r}\right|}=-\frac{r^{2}}{3} R_{k i} x^{k}+O\left(r^{3}\right) \quad \text { for } i=1, \ldots, N .
$$

Moreover, since $\nu_{2}\left(B, h_{r}\right)=r \nu_{2}\left(E\left(y_{0}, r\right), g\right)$, the asserted expansion (45) is equivalent to

$$
\nu_{2}\left(B, h_{r}\right)=1+\frac{2 r^{2}}{3 N(N+2)} S\left(y_{0}\right)+o\left(r^{2}\right) .
$$

Let $\Phi_{r}$ be an eigenfunction for $\nu_{2}\left(B, h_{r}\right)$, normalized such that $\int_{\partial B} \Phi_{r}^{2} d v_{h_{r}}=1$ with $d v_{h_{r}}=$ $\sqrt{\left|h_{r}\right|} d x$. Then we have

$$
\Delta_{h_{r}} \Phi_{r}=0 \quad \text { in } B, \quad\left\langle\nabla \Phi_{r}, \eta\right\rangle_{h_{r}}=\nu_{2}\left(B, h_{r}\right) \Phi_{r} \quad \text { on } \partial B,
$$

where

$$
\Delta_{h_{r}} \Phi_{r}=\frac{1}{\sqrt{\left|h_{r}\right|}} \frac{\partial}{\partial x^{i}}\left(\sqrt{\left|h_{r}\right|} h_{r}^{i j} \frac{\partial \Phi_{r}}{\partial x^{j}}\right) .
$$

Since $h_{r}$ converges to the Euclidean metric in $B$, the variational characterization of $\nu_{2}$ implies that $\nu_{2}\left(B, h_{r}\right) \rightarrow \nu_{2}(B)=1$. Moreover, as in the proof of Proposition 2.2 we have $\Phi_{r_{k}} \rightarrow \Phi$ in $H^{1}(B)$ along a sequence $r_{k} \rightarrow 0$ with some function $\Phi \in C_{l o c}^{2}(B) \cap C^{1}(\bar{B})$ satisfying

$$
\Delta \Phi=0 \quad \text { in } B, \quad\langle\nabla \Phi, \eta\rangle=\Phi \quad \text { on } \partial B, \quad \text { and } \quad \int_{\partial B} \Phi^{2} d \sigma=1 .
$$

Hence there exists a vector $a=\left(a_{1}, \ldots, a_{N}\right)=\left(a^{1}, \ldots, a^{N}\right) \in \mathbb{R}^{N}$ with $|a|=1$ and such that

$$
\Phi(x)=\frac{a \cdot x}{\sqrt{|B|}} \quad \text { for } x \in \bar{B} .
$$

Again, for matters of convenience, we write $r$ instead of $r_{k}$ in the following. By multiple integration by parts, using (49) and (50), we have

$$
\begin{aligned}
\nu_{2}\left(B, h_{r}\right) & \int_{\partial B} \Phi \Phi_{r} d \sigma_{h_{r}}=\int_{B} \sqrt{\mid h_{r}} h_{r}^{i j} \frac{\partial \Phi}{\partial x^{i}} \frac{\partial \Phi_{r}}{\partial x^{j}} d x \\
= & \int_{B} \nabla \Phi_{r} \nabla \Phi d v_{h_{r}}-r^{2} \int_{B}\left(\frac{1}{3} R_{k l}^{i j} x^{k} x^{l} \frac{\partial \Phi_{r}}{\partial x^{i}} \frac{\partial \Phi_{r}}{\partial x^{j}}+2 b^{i} \frac{\partial \Phi}{\partial x^{i}} \frac{\partial \Phi_{r}}{\partial x^{i}}\right) d x+O\left(r^{3}\right) \\
= & \int_{\partial B} \Phi \Phi_{r} d \sigma_{h_{r}}-\int_{B} \Phi_{r} \nabla \sqrt{\left|h_{r}\right|} \cdot \nabla \Phi d x \\
& -r^{2} \int_{B}\left(\frac{1}{3} R_{k l}^{i j} x^{k} x^{l} \frac{\partial \Phi_{r}}{\partial x^{i}} \frac{\partial \Phi_{r}}{\partial x^{j}}+2 b^{i} \frac{\partial \Phi}{\partial x^{i}} \frac{\partial \Phi_{r}}{\partial x^{i}}\right) d x+O\left(r^{3}\right) \\
= & \int_{\partial B} \Phi \Phi_{r} d \sigma_{h_{r}} \\
& +r^{2} \int_{B}\left(\frac{\Phi_{r}}{3} R_{i}{ }^{j} x^{i} \frac{\partial \Phi}{\partial x^{j}}-\frac{1}{3} R_{k l}^{i j} x^{k} x^{l} \frac{\partial \Phi_{r}}{\partial x^{i}} \frac{\partial \Phi_{r}}{\partial x^{j}}-2 b^{i} \frac{\partial \Phi}{\partial x^{i}} \frac{\partial \Phi_{r}}{\partial x^{i}}\right) d x+O\left(r^{3}\right)
\end{aligned}
$$

Since $\int_{\partial B} \Phi \Phi_{r} d \sigma_{h_{r}} \rightarrow 1$ and $\Phi_{r} \rightarrow \Phi$ in $H^{1}(B)$ as $r \rightarrow 0$, we infer, using (36) and (37), that

$$
\begin{aligned}
\nu_{2}\left(B, h_{r}\right) & =1+r^{2} \int_{B}\left(\frac{\Phi}{3} R_{i}{ }^{j} x^{i} \frac{\partial \Phi}{\partial x^{j}}-\frac{1}{3} R_{k l}^{i j} x^{k} x^{l} \frac{\partial \Phi}{\partial x^{i}} \frac{\partial \Phi}{\partial x^{j}}-2 b^{i} \frac{\partial \Phi}{\partial x^{i}} \frac{\partial \Phi}{\partial x^{i}}\right) d x+o\left(r^{2}\right) \\
& =1+r^{2} \int_{B}\left(\frac{1}{3|B|} a_{k} x^{k} R_{i}^{j} a_{j}-\frac{1}{3|B|} R_{k l}^{i j} x^{k} x^{l} a_{i} a_{j}-\frac{2}{|B|} b^{i} a_{i}^{2}\right) d x+o\left(r^{2}\right) \\
& =1+2 r^{2}\left(\frac{1}{3(N+2)} R i c_{y_{0}}(A, A)-b^{i} a_{i}^{2}\right)+o\left(r^{2}\right)
\end{aligned}
$$


with $A:=a^{i} E_{i} \in T_{y_{0}} \mathcal{M}$. Combining this with (43) and (44), we conclude that

$$
\begin{aligned}
\nu_{2}\left(B, h_{r}\right) & =1+2 r^{2}\left(a^{i}\right)^{2}\left(\frac{1}{3(N+2)} R_{i i}-b_{i}\right)+o\left(r^{2}\right) \\
& =1+\frac{2 r^{2}}{3 N(N+2)} S\left(y_{0}\right)+o\left(r^{2}\right) .
\end{aligned}
$$

Hence we have shown (51), as required.

Corollary 2.5 We have

$$
\nu_{2}\left(E\left(y_{0}, r\right), g\right)=\left(\frac{v}{|B|}\right)^{-\frac{1}{N}}+\frac{S\left(y_{0}\right)}{2 N(N+2)}\left(\frac{v}{|B|}\right)^{\frac{1}{N}}+o\left(v^{\frac{1}{N}}\right)
$$

as $v=\left|E\left(y_{0}, r\right)\right|_{g} \rightarrow 0$.

Proof. This follows readily by combining (45) and (46).

\section{A local upper bound for $\nu_{2}$}

The aim of this section is to complete the proof of Theorem 1.1 We note that Theorem 1.1(i) follows immediately from Corollary 2.5, and the lower bound in (ii) is a direct consequence of (i). Hence it remains to establish the upper bound (ii). For this we fix $r_{0}>0$ less than the injectivity radius of $\mathcal{M}$ at $y_{0}$. Throughout this section, we consider a sequence of numbers $r_{k} \in\left(0, \frac{r_{0}}{4}\right)$ such that $r_{k} \rightarrow 0$ as $k \rightarrow \infty$, and we suppose that we are given regular domains $\Omega_{r_{k}} \subset B_{g}\left(y_{0}, r_{k}\right)$, $k \in \mathbb{N}$. In this setting, we will show the following asymptotic upper bound.

Theorem 3.1 We have

$$
\nu_{2}\left(\Omega_{r_{k}}, g\right) \leq\left(\frac{\left|\Omega_{r_{k}}\right|_{g}}{|B|}\right)^{-\frac{1}{N}}+\frac{S\left(y_{0}\right)}{2 N(N-2)}\left(\frac{\left|\Omega_{r_{k}}\right|_{g}}{|B|}\right)^{\frac{1}{N}}+o\left(\left|\Omega_{r_{k}}\right|_{g}^{\frac{1}{N}}\right) \quad \text { as } k \rightarrow \infty .
$$

This result obviously implies the upper bound in Theorem 1.1(ii), so the proof of Theorem 1.1 is finished once we have established Theorem 3.1.

The remainder of this section is devoted to the proof of Theorem 3.1. In order to keep the notation as simple as possible, we will write $r$ instead of $r_{k}$ in the following. As in the previous sections, we rescale the problem, but we first need to identify suitable center points for the rescaling procedure. For this, we need the following observation.

Lemma 3.2 There exists a point $p_{r} \in B_{g}\left(y_{0}, 2 r\right)$ with

$$
\int_{\partial \Omega_{r}} \operatorname{Exp}_{p_{r}}^{-1}(q) d \sigma_{g}(q)=0 \in T_{p_{r}} \mathcal{M} .
$$

Proof. Consider the function

$$
J: \overline{B_{g}\left(y_{0}, 2 r\right)} \rightarrow \mathbb{R}, \quad J(p)=\int_{\partial \Omega_{r}}\left|\operatorname{Exp}_{p}^{-1}(q)\right|_{g}^{2} d \sigma_{g}(q)=\int_{\partial \Omega_{r}} \operatorname{dist}_{g}(p, q)^{2} d \sigma_{g}(q) .
$$

Since $r<r_{0}$ and $\Omega_{r} \subset B_{g}\left(y_{0}, r\right)$, the function $J$ is differentiable with

$$
d J(p)[v]=-2 \int_{\partial \Omega_{r}}\left\langle\operatorname{Exp}_{p}^{-1}(q), v\right\rangle_{g} d \sigma_{g}(q) \quad \text { for all } v \in T_{p} \mathcal{M} .
$$

Since $J\left(y_{0}\right) \leq r^{2} \sigma_{g}\left(\partial \Omega_{r}\right)$ and

$$
J(p) \geq r^{2} \sigma_{g}\left(\partial \Omega_{r}\right) \quad \text { for } p \in \partial B_{g}\left(y_{0}, 2 r\right),
$$


there exists a point $p_{r} \in B_{g}\left(y_{0}, 2 r\right)$ with $J\left(p_{r}\right)=\min \left\{J(p): p \in B_{g}\left(y_{0}, 2 r\right)\right\}$. Hence $p_{r}$ is a critical point of $J$, and this implies (54).

Next we note that, for $r>0$ small enough, we have $\left|B_{g}\left(p_{r}, 2 r\right)\right|_{g}>\left|B_{g}\left(y_{0}, r\right)\right|_{g}$, and thus there exists a unique $\rho_{r} \in(0,2 r)$ with

$$
\left|\Omega_{r}\right|_{g}=\left|B_{g}\left(p_{r}, \rho_{r}\right)\right|_{g} .
$$

Since $p_{r} \rightarrow y_{0}$ as $r \rightarrow 0$, we have, similarly as in (12), the volume expansion

$$
\frac{\left|\Omega_{r}\right|_{g}}{|B|}=\frac{\left|B_{g}\left(p_{r}, \rho_{r}\right)\right|_{g}}{|B|}=\rho_{r}^{N}\left(1-\frac{S\left(y_{0}\right)}{6(N+2)} \rho_{r}^{2}+o\left(\rho_{r}^{2}\right)\right)
$$

and thus

$$
\left(\frac{\left|\Omega_{r}\right|_{g}}{|B|}\right)^{\frac{1}{N}}=\rho_{r}\left(1-\frac{S\left(y_{0}\right)}{6 N(N+2)} \rho_{r}^{2}+o\left(\rho_{r}^{2}\right)\right) .
$$

Consequently, Theorem 3.1 is proved once we establish the following:

$$
\nu_{2}\left(\Omega_{r}, g\right) \leq \frac{1}{\rho_{r}}+\frac{2 \rho_{r}}{3 N(N+2)} S\left(y_{0}\right)+o\left(\rho_{r}\right) \quad \text { as } r \rightarrow 0 .
$$

We now consider a rescaled version of (56). For this we note that

$$
B_{g}\left(p_{r}, \rho_{r}\right) \subset B_{g}\left(p_{r}, 2 r\right) \subset B_{g}\left(y_{0}, 4 r\right) \quad \text { and } \quad \Omega_{r} \subset B_{g}\left(y_{0}, r\right) \subset B_{g}\left(p_{r}, 3 r\right),
$$

and we let

$$
y \mapsto E_{i}^{y} \in T_{y} \mathcal{M}, \quad i=1, \ldots, N
$$

denote a smooth orthonormal frame on $B_{g}\left(y_{0}, r_{0}\right)$. We consider the maps

$$
\Psi_{r}: \mathbb{R}^{N} \rightarrow \mathcal{M}, \quad \Psi_{r}(x)=\Psi\left(\rho_{r} x\right)=\operatorname{Exp}_{p_{r}}\left(\rho_{r} x^{i} E_{i}^{p_{r}}\right) .
$$

Moreover, we set

$$
B^{r}:=\frac{3 r}{\rho_{r}} B \quad \text { and } \quad U_{r}:=\Psi_{r}^{-1}\left(\Omega_{r}\right) \subset B^{r},
$$

and we consider the pull back metric of $g$ under the map $B^{r} \rightarrow \mathcal{M}, x \mapsto \Psi_{r}\left(\rho_{r} x\right)$, rescaled with the factor $\frac{1}{\rho_{r}^{2}}$. We denote this metric on $B^{r}$ by $g_{r}$, and we point out that this definition differs from the notation used in the proof of Proposition 2.2. Nevertheless, since $\operatorname{dist}\left(p_{r}, y_{0}\right)=O(r)$, we have, in $C^{1}$-sense,

$$
\left\langle R_{y}\left(E_{i}^{p_{r}}, E_{j}^{p_{r}}\right) E_{k}^{p_{r}}, E_{l}^{p_{r}}\right\rangle=R_{i j k l}+O(r) \quad \text { as } r \rightarrow 0 \quad \text { with } \quad R_{i j k l}:=\left\langle R_{y_{0}}\left(E_{i}^{y_{0}}, E_{j}^{y_{0}}\right) E_{k}^{y_{0}}, E_{l}^{y_{0}}\right\rangle
$$

for $i, j, k, l=1, \ldots, N$. We also set $R_{i j}:=\operatorname{Ric}_{y_{0}}\left(E_{i}^{y_{0}}, E_{j}^{y_{0}}\right)$. As in Section 2, we freely vary the (upper or lower) position of the indices of $R_{i j k l}$ and $R_{i j}$ without changing the value of these constants. We then infer from (10) and (11) that

$$
\begin{aligned}
\left(g_{r}\right)_{i j}(x) & =\delta_{i j}+\frac{\rho_{r}^{2}}{3} R_{k i l j} x^{k} x^{l}+O\left(r \rho_{r}^{2}\right), \\
g_{r}^{i j}(x) & =\delta^{i j}-\frac{\rho_{r}^{2}}{3} R_{k l}^{i j} x^{k} x^{l}+O\left(r \rho_{r}^{2}\right), \\
d v_{g_{r}}(x)=\sqrt{\left|g_{r}(x)\right|} d x & =\left(1-\frac{\rho_{r}^{2}}{6} R_{l k} x^{l} x^{k}+O\left(r \rho_{r}^{2}\right)\right) d x, \\
\frac{\partial}{\partial x_{i}} \sqrt{\left|g_{r}(x)\right|} & =-\frac{\rho_{r}^{2}}{3} R_{k i} x^{k}+O\left(r \rho_{r}^{2}\right)
\end{aligned}
$$

uniformly on $B^{r}$ as $r \rightarrow 0$, where $\left(g_{r}^{i j}\right)_{i j}$ denotes the inverse of the matrix $\left(\left[g_{r}\right]_{i j}\right)_{i j}$ and $\left|g_{r}\right|$ is the determinant of $g_{r}$. In particular

$$
\left(g_{r}\right)_{i j}(x)=\delta_{i j}+O\left(r^{2}\right) \text { and } \quad d v_{g_{r}}(x)=\left(1+O\left(r^{2}\right)\right) d x \quad \text { uniformly on } B^{r} .
$$


Moreover, by the same arguments as in the proof of Lemma 2.1 we have

$$
\int_{\partial U_{r}} f(x) d \sigma_{g_{r}}=\left(1+O\left(\rho_{r}^{2}\right)\right) \int_{\partial U_{r}} f(x) d \sigma
$$

for every $f \in C^{1}\left(\partial U_{r}\right)$. Moreover,

$$
\int_{\partial B} f(x) d \sigma_{g_{r}}(x)=\int_{\partial B}\left(1-\frac{\rho_{r}^{2}}{6} R_{l k} x^{l} x^{k}\right) f(x) d \sigma+O\left(r \rho_{r}^{2}\right) \int_{\partial B} f(x) d \sigma
$$

for every $f \in C^{1}(\partial B)$. Here, similarly as in Lemma 2.1, the bounds for the terms $O\left(\rho_{r}^{2}\right)$ and $O\left(r \rho_{r}^{2}\right)$ are uniform in $f$. Observe also that $\nu_{2}\left(U_{r}, g_{r}\right)=\frac{\nu_{2}\left(\Omega_{r}, g\right)}{\rho_{r}}$, so that (56) is equivalent to

$$
\nu_{2}\left(U_{r}, g_{r}\right) \leq 1+\frac{2 \rho_{r}^{2}}{3 N(N+2)} S\left(y_{0}\right)+o\left(\rho_{r}^{2}\right) \quad \text { as } r \rightarrow 0 .
$$

The remainder of this section will be devoted to the proof of (62). By construction we have $\left|U_{r}\right|_{g_{r}}=\rho_{r}{ }^{-N}\left|\Omega_{r}\right|_{g}=\rho_{r}{ }^{-N}\left|B_{g}\left(p_{r}, \rho_{r}\right)\right|_{g}=|B|_{g_{r}}$, and thus

$$
\left|U_{r}\right|_{g_{r}}=|B|_{g_{r}}=\left(1+O\left(r^{2}\right)\right)\left|U_{r}\right|=\left(1+O\left(r^{2}\right)\right)|B|
$$

by (59) and the fact that $U_{r} \subset B^{r}$ and $B \subset B^{r}$. Setting

$$
f_{i}: \mathbb{R}^{N} \rightarrow \mathbb{R}, \quad f_{i}(x)=x^{i}
$$

we also find that $\int_{\partial U_{r}} f_{i} d \sigma_{g_{r}}=0$ for $i=1, \ldots, N$ by (54). Moreover,

$$
\int_{U_{r}}\left|\nabla f_{i}\right|_{g_{r}}^{2} d v_{g_{r}}=\int_{U_{r}} g_{r}^{j k} \frac{\partial f_{i}}{\partial x^{j}} \frac{\partial f_{i}}{\partial x^{k}} d v_{g_{r}}=\int_{U_{r}} g_{r}^{i i} d v_{g_{r}} \quad \text { for } i=1, \ldots, N .
$$

Hence the variational characterization of $\nu_{2}$ yields

$$
\nu_{2}\left(U_{r}, g_{r}\right) \leq \frac{\sum_{i=1}^{N} \int_{U_{r}}\left|\nabla f_{i}\right|_{g_{r}}^{2} d v_{g_{r}}}{\sum_{i=1}^{N} \int_{\partial U_{r}} f_{i}^{2} d \sigma_{g_{r}}}=\frac{\sum_{i=1}^{N} \int_{U_{r}} g_{r}^{i i} d v_{g_{r}}}{\int_{\partial U_{r}}|x|^{2} d \sigma_{g_{r}}}
$$

In the following, $B_{2}:=2 B \subset \mathbb{R}^{N}$ denotes the euclidean ball centered at the origin with radius 2 . Moreover, we let $\left|U_{r} \triangle B\right|=\left|U_{r} \backslash B\right|+\left|B \backslash U_{r}\right|$ denote the symmetric distance of the sets $U_{r}$ and $B$ with respect to the standard Lebesgue measure on $\mathbb{R}^{N}$.

Lemma 3.3 In the above setting, we have

$$
\int_{\partial U_{r}}|x|^{2} d \sigma_{g_{r}} \geq N|B|-\frac{|B| \rho_{r}^{2}}{6} S\left(y_{0}\right)+O\left(\rho_{r}^{2}\left|U_{r} \triangle B\right|\right)+\frac{N+1}{4}\left|U_{r} \backslash B_{2}\right|_{g_{r}}+o\left(\rho_{r}^{2}\right)
$$

and

$$
\sum_{i=1}^{N} \int_{U_{r}} g_{r}^{i i} d v_{g_{r}}=N|B|-\frac{(N-2)|B|}{6(N+2)} \rho_{r}^{2} S\left(y_{0}\right)+O\left(\rho_{r}^{2}\left|U_{r} \triangle B\right|\right)+O\left(r^{2}\left|U_{r} \backslash B_{2}\right| g_{r}\right)+o\left(\rho_{r}^{2}\right)
$$

as $r \rightarrow 0$.

Proof. We first note that, by (59), the symmetric distance $\left|U_{r} \triangle B\right|_{g_{r}}:=\left|U_{r} \backslash B\right|_{g_{r}}+\left|B \backslash U_{r}\right|_{g_{r}}$ with respect to the metric $g_{r}$ satisfies

$$
\left|U_{r} \triangle B\right|_{g_{r}}=\left(1+O\left(r^{2}\right)\right)\left|U_{r} \triangle B\right|
$$


Next we consider the $C^{1}$-vector field $V: B^{r} \rightarrow \mathbb{R}, V(x)=|x| x$. Using (158), we have

$$
\begin{aligned}
G(x) & :=\operatorname{div}_{g_{r}}(V)=\frac{1}{\sqrt{\left|g_{r}\right|}(x)} \frac{\partial}{\partial x^{i}}\left(|x| x^{i} \sqrt{\left|g_{r}\right|}(x)\right)=|x|\left[(N+1)-\frac{x \cdot \nabla \sqrt{\left|g_{r}\right|(x)}}{\sqrt{\left|g_{r}\right|(x)}}\right] \\
& =|x|\left[(N+1)-\frac{\frac{\rho_{r}^{2}}{3} \operatorname{Ric}_{y_{0}}(X, X)+O\left(r \rho_{r}^{2}\right)}{\sqrt{\left|g_{r}\right|(x)}}\right]=|x|\left[(N+1)-\frac{\rho_{r}^{2}}{3} \operatorname{Ric}_{y_{0}}(X, X)+O\left(r \rho_{r}^{2}\right)\right] \\
& =|x|\left[(N+1)-\frac{\rho_{r}^{2}}{3} \operatorname{Ric}_{y_{0}}(X, X)+O\left(r \rho_{r}^{2}\right)\right] \quad \text { uniformly for } x \in B^{r} \text { as } r \rightarrow 0 .
\end{aligned}
$$

In particular, $G(x)=|x|\left[(N+1)+O\left(r^{2}\right)\right]$ for $x \in B^{r}$ as $r \rightarrow 0$, so for $r>0$ sufficiently small we have

$$
G(x) \geq \frac{N+1}{2}|x| \quad \text { for } x \in B^{r} .
$$

We also recall that, as a consequence of Gauss' Lemma (see e.g. [11, Corollary 5.2.3]), the unit outer normal on $\partial B$ with respect to the metric $g_{r}$ is simply given by $\eta_{r}(x)=x$ for every small $r>0$ and $x \in \partial B$. Using the divergence formula with respect to the metric $g_{r}$ and (61), we therefore find that

$$
\int_{B} G d v_{g_{r}}=\int_{\partial B} d \sigma_{g_{r}}=\int_{\partial B}\left(1-\frac{\rho_{r}^{2}}{6} R_{l k} x^{l} x^{k}\right) d \sigma+O\left(r \rho_{r}^{2}\right)=N|B|-\frac{|B| \rho_{r}^{2}}{6} S\left(y_{0}\right)+o\left(\rho_{r}^{2}\right) .
$$

Here we used the fact that $\int_{\partial B} x^{l} x^{k} d \sigma=\delta^{l k} \frac{\sigma(\partial B)}{N}=\delta^{l k}|B|$ in the last step. Moreover, using again that $|x|_{g_{r}}=|x|$ and thus $|V(x)|_{g_{r}}=|x|^{2}$ for $x \in B^{r}$ by Gauss' Lemma, we find that

$$
\int_{U_{r}} G d v_{g_{r}}=\int_{\partial U_{r}}\left\langle V, \eta_{r}\right\rangle_{g_{r}} d \sigma_{g_{r}} \leq \int_{\partial U_{r}}|V|_{g_{r}} d \sigma_{g_{r}}=\int_{\partial U_{r}}|x|^{2} d \sigma_{g_{r}},
$$

where $\eta_{r}$ is the outer unit normal of $\partial U_{r}$ with respect to $g_{r}$. Next we estimate

$$
\begin{aligned}
\int_{U_{r}} G d v_{g_{r}} & -\int_{B} G d v_{g_{r}}=\int_{U_{r} \backslash B} G d v_{g_{r}}-\int_{B \backslash U_{r}} G d v_{g_{r}} \\
& =\int_{U_{r} \backslash B}\left(1-\frac{1}{|x|}\right) G d v_{g_{r}}+\int_{U_{r} \backslash B} \frac{G}{|x|} d v_{g_{r}}-\int_{B \backslash U_{r}} G d v_{g_{r}} \\
& \geq \int_{U_{r} \backslash B}\left(1-\frac{1}{|x|}\right) G d v_{g_{r}}+\int_{U_{r} \backslash B} \frac{G}{|x|} d v_{g_{r}}-\int_{B \backslash U_{r}} \frac{G}{|x|} d v_{g_{r}} .
\end{aligned}
$$

Here we note that, by (69),

$$
\int_{U_{r} \backslash B}\left(1-\frac{1}{|x|}\right) G d v_{g_{r}} \geq \int_{U_{r} \backslash B_{2}}\left(1-\frac{1}{|x|}\right) G d v_{g_{r}} \geq \int_{U_{r} \backslash B_{2}} \frac{G}{|x|} d v_{g_{r}} \geq \frac{N+1}{2}\left|U_{r} \backslash B_{2}\right|_{g_{r}}
$$

and, by (63) and (68),

$$
\begin{aligned}
\int_{U_{r} \backslash B} \frac{G}{|x|} d v_{g_{r}} & -\int_{B \backslash U_{r}} \frac{G}{|x|} d v_{g_{r}}=\int_{U_{r} \backslash B}\left(\frac{G}{|x|}-(N+1)\right) d v_{g_{r}}-\int_{B \backslash U_{r}}\left(\frac{G}{|x|}-(N+1)\right) d v_{g_{r}} \\
& =\frac{\rho_{r}^{2}}{3}\left[\int_{B \backslash U_{r}}\left(R_{i j} x^{i} x^{j}+O(r)\right) d v_{g_{r}}-\int_{U_{r} \backslash B}\left(R_{i j} x^{i} x^{j}+O(r)\right) d v_{g_{r}}\right] \\
& =\frac{\rho_{r}^{2}}{3}\left[\int_{B \backslash U_{r}} R_{i j} x^{i} x^{j} d v_{g_{r}}-\int_{U_{r} \backslash B} R_{i j} x^{i} x^{j} d v_{g_{r}}+O\left(r\left|B \triangle U_{r}\right|_{g_{r}}\right)\right] \\
& =\frac{\rho_{r}^{2}}{3}\left[-\int_{U_{r} \backslash B_{2}} R_{i j} x^{i} x^{j} d v_{g_{r}}+O\left(\left|B \triangle U_{r}\right|_{g_{r}}\right)\right] \\
& =O\left(r^{2}\left|U_{r} \backslash B_{2}\right|_{g_{r}}\right)+O\left(\rho_{r}^{2}\left|U_{r} \triangle B\right|\right)+o\left(\rho_{r}^{2}\right),
\end{aligned}
$$


where in the last step we used (67) and the fact that $U_{r} \subset B^{r}$. Combining (72), (73) and (74), we obtain that

$$
\int_{U_{r}} G d v_{g_{r}} \geq \int_{B} G d v_{g_{r}}+O\left(\rho_{r}^{2}\left|U_{r} \triangle B\right|\right)+\frac{N+1}{4}\left|U_{r} \backslash B_{2}\right|_{g_{r}} \quad \text { for } r>0 \text { sufficiently small. }
$$

Combining this with (70) and (71), we get the inequality

$$
\int_{\partial U_{r}}|x|^{2} d \sigma_{g_{r}} \geq N|B|-\frac{|B| \rho_{r}^{2}}{6} S\left(y_{0}\right)+\frac{N+1}{4}\left|U_{r} \backslash B_{2}\right|_{g_{r}}+O\left(\rho_{r}^{2}\left|U_{r} \triangle B\right|\right)+o\left(\rho_{r}^{2}\right)
$$

as $r \rightarrow 0$, which is (65). Next, using (63), we estimate similarly as in (74),

$$
\begin{aligned}
& \sum_{i=1}^{N} \int_{U_{r}} g_{r}^{i i} d v_{g_{r}}=\int_{U_{r}}\left[N+\frac{\rho_{r}^{2}}{3} R_{i j} x^{i} x^{j}+O\left(r \rho_{r}^{2}\right)\right] d v_{g_{r}} \\
& =\int_{B}\left(N+\frac{\rho_{r}^{2}}{3} R_{i j} x^{i} x^{j}+o\left(\rho_{r}^{2}\right)\right) d v_{g_{r}}+\frac{\rho_{r}^{2}}{3} \int_{U_{r} \backslash B_{2}}\left(R_{i j} x^{i} x^{j}+O(r)\right) d v_{g_{r}}+O\left(\rho_{r}^{2}\left|U_{r} \triangle B\right|_{g_{r}}\right) \\
& =N|B|-\frac{(N-2)|B|}{6(N+2)} \rho_{r}^{2} S\left(y_{0}\right)+o\left(\rho_{r}^{2}\right)+O\left(r^{2}\left|U_{r} \backslash B_{2}\right| g_{r}\right)+O\left(\rho_{r}^{2}\left|U_{r} \triangle B\right|_{g_{r}}\right) \\
& =N|B|-\frac{(N-2)|B|}{6(N+2)} \rho_{r}^{2} S\left(y_{0}\right)+O\left(r\left|U_{r} \backslash B_{2}\right|_{g_{r}}\right)+O\left(\rho_{r}^{2}\left|U_{r} \triangle B\right|\right)+o\left(\rho_{r}^{2}\right)
\end{aligned}
$$

as $r \rightarrow 0$, as claimed in (66).

We may now complete the

Proof of Theorem 3.1. As noted before, it suffices to prove (62), since (62) is equivalent to (56) and (56) is equivalent to (53) by the volume expansion (55). To prove (62) for $r=r_{k} \rightarrow 0$ as $k \rightarrow \infty$, we argue by contradiction and assume that there exists $\varepsilon_{0}>0$ and a subsequence - still denoted by $\left(r_{k}\right)_{k}-$ such

$$
\nu_{2}\left(U_{r_{k}}, g_{r_{k}}\right) \geq 1+\left(\frac{2}{3 N(N+2)} S\left(y_{0}\right)+\varepsilon_{0}\right) \rho_{r_{k}}^{2} \quad \text { for all } k \in \mathbb{N} .
$$

We first claim that

$$
\left|U_{r_{k}} \backslash B_{2}\right|_{g_{r_{k}}}=O\left(\rho_{r_{k}}^{2}\right) \quad \text { as } k \rightarrow \infty .
$$

Indeed, if, by contradiction, for a subsequence we have $\left|U_{r_{k}} \backslash B_{2}\right|_{g_{r_{k}}} \geq k \rho_{r_{k}}^{2}$, then the expansions (65) and (66) yield that

$$
\int_{\partial U_{r_{k}}}|x|^{2} d \sigma_{g_{r_{k}}} \geq \sum_{i=1}^{N} \int_{U_{r_{k}}} g_{r_{k}}^{i i} d v_{g_{r_{k}}} \quad \text { for } k \text { sufficiently large. }
$$

Here we also used the fact $\left|U_{r_{k}} \triangle B\right|$ remains bounded as a consequence of (63). Now (64) implies that $\nu_{2}\left(U_{r_{k}}, g_{r_{k}}\right) \leq 1$ for $k$ sufficiently large, contrary to (76). Hence (77) is true. From (77) and (66) it then follows that

$$
\sum_{i=1}^{N} \int_{U_{r_{k}}} g_{r_{k}}^{i i} d v_{g_{r_{k}}}=N|B|+O\left(r_{k}^{2}\right)
$$

Since also $\nu_{2}\left(U_{r_{k}}, g_{r_{k}}\right) \geq 1+O\left(r_{k}^{2}\right)$ by (76), it follows from (64) that

$$
\int_{\partial U_{r_{k}}}|x|^{2} d \sigma_{g_{r_{k}}} \leq N|B|+O\left(r_{k}^{2}\right) \text {. }
$$

and thus also

$$
\int_{\partial U_{r_{k}}}|x|^{2} d \sigma \leq N|B|+O\left(r_{k}^{2}\right)
$$


as a consequence of (60). On the other hand, [1, Theorem B] implies that

$$
\int_{\partial U_{r_{k}}}|x|^{2} d \sigma \geq N|B|+\beta\left(\frac{\left|U_{r_{k}} \triangle B\right|}{\left|U_{r_{k}}\right|}\right)^{2}
$$

with a positive constant $\beta>0$. Hence, by (63) and (79),

$$
\left|U_{r_{k}} \triangle B\right|=O\left(\left|U_{r_{k}}\right| r_{k}{ }^{2}\right)=O\left(r_{k}^{2}\right) \quad \text { as } k \rightarrow \infty .
$$

Inserting this in (65) gives

$$
\int_{\partial U_{r_{k}}}|x|^{2} d \sigma_{g_{r_{k}}} \geq N|B|-\frac{|B|}{6} S\left(y_{0}\right) \rho_{r_{k}}^{2}+o\left(\rho_{r_{k}}^{2}\right) .
$$

Moreover, inserting (77) and (81) in (66) gives

$$
\sum_{i=1}^{N} \int_{U_{r_{k}}} g_{r_{k}}^{i i} d v_{g_{r_{k}}}=N|B|-\frac{(N-2)|B|}{6(N+2)} S\left(y_{0}\right) \rho_{r_{k}}^{2}+o\left(\rho_{r_{k}}^{2}\right) .
$$

Combining (82), (83) and (64) finally yields

$$
\nu_{2}\left(U_{r_{k}}, g_{r_{k}}\right) \leq 1+\frac{2 \rho_{r_{k}}^{2}}{3 N(N+2)} S\left(y_{0}\right)+o\left(\rho_{r_{k}}^{2}\right) \quad \text { as } k \rightarrow \infty,
$$

contrary to (76). The proof is finished.

\section{Precise global asymptotics in the two-dimensional case}

In this section we give the proof of Theorem 1.2. We shall see that most of the argument works for $N \geq 2$ except at the end of the proof of Lemma 4.2 below where we had to assume that $N=2$. For convenience, we repeat the statement of the theorem.

Theorem 4.1 Let $(\mathcal{M}, g)$ be a closed Riemannian surface. Then we have

$$
W B_{\mathcal{M}}(v)=\left(\frac{v}{\pi}\right)^{-\frac{1}{2}}+\frac{S_{\mathcal{M}}}{16}\left(\frac{v}{\pi}\right)^{\frac{1}{2}}+o\left(v^{\frac{1}{2}}\right) \quad \text { as } v \rightarrow 0,
$$

where $S_{\mathcal{M}}$ denotes the maximum of the scalar curvature function $S$ on $\mathcal{M}$.

The remainder of this section is devoted to the proof of this result. In view of Theorem 1.1(i) and the remarks after this theorem, we only need to prove that

$$
W B_{\mathcal{M}}(v) \leq\left(\frac{v}{\pi}\right)^{-\frac{1}{2}}+\frac{S_{\mathcal{M}}}{16}\left(\frac{v}{\pi}\right)^{\frac{1}{2}}+o\left(v^{\frac{1}{2}}\right) \quad \text { as } v \rightarrow 0 .
$$

We argue by contradiction and assume that there exists $\bar{\varepsilon}>0$ and a sequence of regular domains $\Omega_{k} \subset \mathcal{M}$ such that $v_{k}:=\left|\Omega_{k}\right| \rightarrow 0$ as $k \rightarrow \infty$ and

$$
\nu_{2}\left(\Omega_{k}, g\right) \geq\left(\frac{v_{k}}{\pi}\right)^{-\frac{1}{2}}+\left[\frac{S_{\mathcal{M}}}{16}+\bar{\varepsilon}\right]\left(\frac{v_{k}}{\pi}\right)^{\frac{1}{2}} \quad \text { for every } k \in \mathbb{N} .
$$

We will show that

$$
\operatorname{diam}\left(\Omega_{k}\right) \rightarrow 0 \quad \text { as } k \rightarrow \infty .
$$

Once this fact is established, we arrive at a contradiction as follows. By the compactness of $\mathcal{M}$, there exists $y_{0} \in \mathcal{M}$ such that, after passing to a subsequence,

for every $r>0$ there exists $k_{r} \in \mathbb{N}$ such that $\Omega_{k} \subset B_{g}\left(y_{0}, r\right)$ for $k \geq k_{r}$. 
Fix $\varepsilon<\bar{\varepsilon}$, and let $r_{\varepsilon}$ be given by Theorem 1.1(ii) corresponding to these choices of $y_{0}$ and $\varepsilon$. Then, for $k \geq k_{r_{\varepsilon}}$, we have

$$
\nu_{2}\left(\Omega_{k}, g\right) \leq W B_{\mathcal{M}}\left(v_{k}\right) \leq\left(\frac{v_{k}}{\pi}\right)^{-\frac{1}{2}}+\left[\frac{S\left(y_{0}\right)}{16}+\varepsilon\right]\left(\frac{v_{k}}{\pi}\right)^{\frac{1}{2}} \leq\left(\frac{\pi}{v_{k}}\right)^{\frac{1}{2}}+\left[\frac{S_{\mathcal{M}}}{16}+\varepsilon\right]\left(\frac{v_{k}}{\pi}\right)^{\frac{1}{2}}
$$

as a consequence of the upper estimate in Theorem 1.1(ii). This contradicts (85), since $\varepsilon<\bar{\varepsilon}$, and thus the proof of Theorem 4.1 is finished. Hence it remains to prove (86), and the remainder of this section is devoted to this task. Since $\mathcal{M}$ is closed, it is easy to see that there exists a number $K>0$ such that

$$
\text { for every } r>0, p \in \mathcal{M} \text { there exist } p_{1}, \ldots, p_{K} \in \mathcal{M} \text { with } B_{g}(p, 4 r) \subset \bigcup_{i=1}^{K} B_{g}\left(p_{k}, r\right) \text {. }
$$

To prove (86), we now argue by contradiction and assume that there exists $d>0$ such that, after passing to a subsequence, $\operatorname{diam}\left(\Omega_{k}\right) \geq d$ for all $k \in \mathbb{N}$. In the following, we let $r_{\mathcal{M}}$ denote the injectivity radius of $\mathcal{M}$, and we put $r_{0}:=\min \left\{\frac{r_{\mathcal{M}}}{5}, \frac{d}{7}\right\}$. We also let $\varphi \in C_{0}^{\infty}(\mathbb{R}, \mathbb{R})$ be a function such that

$\varphi \equiv 2$ on $(-\infty, 0], \varphi\left(r_{0}^{2}\right)=1, \varphi\left(\left(2 r_{0}\right)^{2}\right)=\frac{1}{K+1}, \varphi^{\prime}<0$ on $\left(0,\left(4 r_{0}\right)^{2}\right)$ and $\varphi \equiv 0$ on $\left[\left(4 r_{0}\right)^{2}, \infty\right)$,

where $K$ has the property in (87). For $p \in \mathcal{M}$ and $v \in T_{p} \mathcal{M}$ we consider the function

$$
f_{p, v} \in C^{\infty}(\mathcal{M}), \quad f_{p, v}(q)= \begin{cases}\varphi^{\prime}\left(\operatorname{dist}(p, q)^{2}\right)\left\langle\operatorname{Exp}_{p}^{-1}(q), v\right\rangle, & q \in B_{g}\left(p, 4 r_{0}\right) \\ 0, & q \notin B_{g}\left(p, 4 r_{0}\right)\end{cases}
$$

Since $\mathcal{M}$ is compact, we find that

$$
c_{0}:=\sup \left\{\left|\nabla f_{p, v}(q)\right|_{g}: q, p \in \mathcal{M}, v \in T_{p} \mathcal{M},|v|_{g}=1\right\}<\infty .
$$

Lemma 4.2 There exists points $p_{k} \in \mathcal{M}$ and vectors $v_{k} \in T_{p_{k}} \mathcal{M}, k \in \mathbb{N}$ with $\left|v_{k}\right|_{g}=1$ and the following properties:

(i) $\partial \Omega_{k} \cap B_{g}\left(p_{k}, 2 r_{0}\right) \neq \varnothing$ for all $k \in \mathbb{N}$.

(ii) Setting $f_{k}:=f_{p_{k}, v_{k}} \in C^{\infty}(\mathcal{M})$, we have $\int_{\partial \Omega_{k}} f_{k} d \sigma_{g}=0$ for all $k \in \mathbb{N}$. Moreover,

$$
c_{1}:=\liminf _{k \rightarrow \infty} \int_{\partial \Omega_{k}} f_{k}^{2} d \sigma_{g}>0 .
$$

Proof. We fix $k \in \mathbb{N}$ and consider the functional

$$
J: \mathcal{M} \rightarrow \mathbb{R}, \quad J(p)=\int_{\partial \Omega_{k}} \varphi\left(\operatorname{dist}(p, q)^{2}\right) d \sigma_{g}(q) .
$$

Since $\mathcal{M}$ is compact, there exists a point $p_{k} \in \mathcal{M}$ such that $J\left(p_{k}\right)=\max _{\mathcal{M}} J$. We claim that

$$
\operatorname{dist}\left(p, \partial \Omega_{k}\right)<2 r_{0} \text {. }
$$

Indeed, suppose by contradiction that $\operatorname{dist}\left(p_{k}, \partial \Omega_{k}\right) \geq 2 r_{0}$. Then

$$
J\left(p_{k}\right) \leq \varphi\left(\left[2 r_{0}\right]^{2}\right) \sigma_{g}\left(\partial \Omega_{k} \cap\left[B_{g}\left(p_{k}, 4 r_{0}\right) \backslash B_{g}\left(p_{k}, 2 r_{0}\right)\right]\right) \leq \frac{\sigma_{g}\left(\partial \Omega_{k} \cap B_{g}\left(p_{k}, 4 r_{0}\right)\right)}{K+1} .
$$

On the other hand, by (87) there exists a point $\bar{p} \in \mathcal{M}$ such that $\sigma_{g}\left(\partial \Omega_{k} \cap B_{g}\left(\bar{p}, r_{0}\right)\right) \geq$ $\frac{\sigma_{g}\left(\partial \Omega_{k} \cap B_{g}\left(p_{k}, 4 r_{0}\right)\right)}{K}$, and thus

$$
J(\bar{p}) \geq \varphi\left(r_{0}^{2}\right) \sigma_{g}\left(\partial \Omega_{k} \cap B_{g}\left(\bar{p}, r_{0}\right)\right) \geq \frac{\sigma_{g}\left(\partial \Omega_{k} \cap B_{g}\left(p_{k}, 4 r_{0}\right)\right)}{K}>J\left(p_{k}\right),
$$


contradiction. Hence (90) is true, and thus (i) follows. By the maximization property of $p_{k}$, we have

$0=d J\left(p_{k}\right)[v]=-2 \int_{\partial \Omega_{k}} \varphi^{\prime}\left(\operatorname{dist}\left(p_{k}, q\right)^{2}\right)\left\langle\operatorname{Exp}_{p_{k}}^{-1}(q), v\right\rangle_{g} d \sigma_{g}=-2 \int_{\partial \Omega_{k}} f_{p_{k}, v} d \sigma_{g} \quad$ for all $v \in T_{p_{k}} \mathcal{M}$,

hence the first part of (ii) follows independently of the choice of $v_{k}$. To prove (89) for suitable $v_{k} \in T_{p_{k}} \mathcal{M}$ with $\left|v_{k}\right|_{g}=1$, we choose orthonormal vectors $v_{k_{1}}, v_{k_{2}} \in T_{p_{k}} \mathcal{M}$ (with respect to $g$ ) for every $k \in \mathbb{N}$. With $\kappa:=\inf \left\{\left[\varphi^{\prime}\right]^{2}(r): r \in\left[2 r_{0}, 3 r_{0}\right]\right\}>0$ and

$$
\Gamma_{k}:=\partial \Omega_{k} \cap\left[B_{g}\left(p_{k}, 3 r_{0}\right) \backslash \overline{B_{g}\left(p_{k}, 2 r_{0}\right)}\right] \quad \text { for } k \in \mathbb{N},
$$

we then have

$$
\begin{aligned}
\sum_{i=1}^{2} \int_{\partial \Omega_{k}} f_{p_{k}, v_{k_{i}}}^{2} d \sigma_{g} & \geq \kappa \sum_{i=1}^{2} \int_{\Gamma_{k}}\left\langle\operatorname{Exp}_{p_{k}}^{-1}(q), v_{k_{i}}\right\rangle^{2} d \sigma_{g}(q)=\kappa \int_{\Gamma_{k}}\left|\operatorname{Exp}_{p_{k}}^{-1}(q)\right|_{g}^{2} d \sigma_{g}(q) \\
& =\kappa \int_{\Gamma_{k}} \operatorname{dist}^{2}\left(p_{k}, q\right) d \sigma_{g}(q) \geq\left(2 r_{0}\right)^{2} \kappa \sigma_{g}\left(\Gamma_{k}\right) .
\end{aligned}
$$

It now remains to show that

$$
\liminf _{k \rightarrow \infty} \sigma_{g}\left(\Gamma_{k}\right)>0 .
$$

Indeed, once (92) is established, we may combine it with (91) to see that, without loss of generality,

$$
\liminf _{k \rightarrow \infty} \int_{\partial \Omega_{k}} f_{p_{k}, v_{k}}^{2} d \sigma_{g}>0 \quad \text { with } v_{k}:=v_{k, 1} \text { for } k \in \mathbb{N} .
$$

Hence (89) holds, and the proof is then finished. To show (92), we put $S_{r, k}:=\partial B_{g}\left(p_{k}, r\right)$ for $k \in \mathbb{N}, r>0$. Since $\operatorname{diam}\left(\Omega_{k}\right) \geq d>6 r_{0}$, the domain $\Omega_{k}$ is not contained in $B_{g}\left(p_{k}, 3 r_{0}\right)$. Hence, by (i) and since $\Omega_{k}$ is connected, we have

$$
\Omega_{k} \cap S_{r, k} \neq \varnothing \quad \text { for every } r \in\left(2 r_{0}, 3 r_{0}\right) .
$$

Next, we let

$$
T_{k}:=\left\{r \in\left(2 r_{0}, 3 r_{0}\right): S_{r, k} \subset \Omega_{k}\right\} \quad \text { and } \quad R_{k}:=\left(2 r_{0}, 3 r_{0}\right) \backslash T_{k} .
$$

We claim that, after passing to a subsequence,

$$
\left|T_{k}\right| \rightarrow 0 \quad \text { as } k \rightarrow \infty .
$$

To see this, we may, by the compactness of $\mathcal{M}$, pass to a subsequence such that $p_{k} \rightarrow p_{0} \in \mathcal{M}$ and $p_{k} \in B_{g}\left(p_{0}, r_{0}\right)$ for all $k \in \mathbb{N}$. We let

$$
y \mapsto E_{i}^{y} \in T_{y} \mathcal{M}, \quad i=1, \ldots, N
$$

denote a smooth orthonormal frame on $B_{g}\left(p_{0}, r_{0}\right)$, and we consider the maps

$$
\Psi_{k}: \mathbb{R}^{N} \rightarrow \mathcal{M}, \quad \Psi_{k}(x)=\operatorname{Exp}_{p_{k}}\left(x^{i} E_{i}^{p_{k}}\right) \quad \text { for } k \in \mathbb{N} \cup\{0\} .
$$

We note that $\Psi_{k}$ converges locally uniformly in $C^{1}$-sense to $\Psi_{0}$ as $k \rightarrow \infty$. Moreover, since $r_{\mathcal{M}} \geq 5 r_{0}, \Psi_{k}$ maps $3 r_{0} B$ diffeomorpically onto $B_{g}\left(p_{0}, 3 r_{0}\right)$ for every $k \in \mathbb{N} \cup\{0\}$, and there exists a constant $\alpha>1$ such that

$$
\frac{1}{\alpha} \leq \sqrt{\operatorname{det}\left(g_{i j}^{k}(x)\right)_{i j}} \leq \alpha \quad \text { for every } x \in 3 r_{0} B, k \in \mathbb{N} \cup\{0\} .
$$

Here $g_{i j}^{k}$ denote the metric coefficients associated with local parametrizations $\Psi_{k}$, i.e.,

$$
g_{i j}^{k}(x)=\left\langle d \Psi_{k}(x) e_{i}, d \Psi_{k}(x) e_{j}\right\rangle_{g} \quad \text { for } x \in \mathbb{R}^{N}, i, j=1, \ldots, N,
$$


where $e_{i} \in \mathbb{R}^{N}, i=1, \ldots, n$ denote the coordinate vectors. We set $U_{k}:=\Psi_{k}^{-1}\left(\Omega_{k} \cap B_{g}\left(p_{k}, 3 r_{0}\right)\right)$ for $k \in \mathbb{N}$. Since $\left|\Omega_{k}\right|_{g} \rightarrow 0$ as $k \rightarrow \infty$, we also have that $\left|U_{k}\right| \rightarrow 0$ as $k \rightarrow \infty$ as a consequence of (95). Moreover, $T_{k}$ is given as the set of $r \in\left(2 r_{0}, 3 r_{0}\right)$ such that $x \in U_{k}$ for every $x \in \mathbb{R}^{N}$ with $|x|=r$. Hence we estimate that

$$
\left|U_{k}\right| \geq \omega_{N-1} \int_{2 r_{0}}^{3 r_{0}} r^{N-1} 1_{T_{k}}(r) d r \geq \omega_{N-1}\left(2 r_{0}\right)^{N-1}\left|T_{k}\right| .
$$

where $\omega_{N-1}$ denotes the euclidean surface measure of the unit sphere in $\mathbb{R}^{N}$. Thus (94) holds, as claimed. From (94) we deduce that

$$
\left|R_{k}\right| \rightarrow r_{0} \quad \text { as } k \rightarrow \infty .
$$

Moreover,

$$
S_{r, k} \cap \partial \Omega_{k} \neq \varnothing \quad \text { for every } r \in R_{k}
$$

as a consequence of (93). Next we claim that

$$
\sigma_{g}\left(\Gamma_{k}\right) \geq\left|R_{k}\right| \quad \text { for every } k \in \mathbb{N} .
$$

Here we shall need the assumption $N=2$. To derive (98), we fix $k \in \mathbb{N}, \varepsilon \in\left(0, \frac{r_{0}}{2}\right)$ and set

$$
\Gamma_{k, \varepsilon}:=\left\{x \in \partial \Omega_{k}: 2 r_{0}+\varepsilon \leq \operatorname{dist}\left(x, p_{k}\right) \leq 3 r_{0}-\varepsilon\right\} \subset \Gamma_{k}
$$

Since $\Omega_{k}$ is smooth and $\Gamma_{k, \varepsilon}$ is compact, only finitely many (disjoint) path components $\Gamma_{k}^{1}, \ldots, \Gamma_{k}^{m}$ of $\Gamma_{k}$ intersect $\Gamma_{k, \varepsilon}$. Let

$$
\beta_{j}^{+}:=\max \left\{\operatorname{dist}\left(p_{k}, q\right): q \in \Gamma_{k}^{j} \cap \Gamma_{k, \varepsilon}\right\} \quad \text { and } \quad \beta_{j}^{-}:=\min \left\{\operatorname{dist}\left(p_{k}, q\right): q \in \Gamma_{k}^{j} \cap \Gamma_{k, \varepsilon}\right\}
$$

for $j=1, \ldots, k$. By construction and (97) we then have

$R_{k} \cap\left[2 r_{0}+\varepsilon, 3 r_{0}-\varepsilon\right] \subset\left\{r \in\left[2 r_{0}+\varepsilon, 3 r_{0}-\varepsilon\right]: \operatorname{dist}\left(x, p_{k}\right)=r \quad\right.$ for some $x \in \Gamma_{k}^{j} \cap \Gamma_{k, \varepsilon}$ and some $\left.j\right\}$

$$
\subset\left\{r \in\left[2 r_{0}+\varepsilon, 3 r_{0}-\varepsilon\right]: \beta_{j}^{-} \leq r \leq \beta_{j}^{+} \text {for some } j\right\}
$$

and therefore

$$
\left|R_{k} \cap\left[2 r_{0}+\varepsilon, 3 r_{0}-\varepsilon\right]\right| \leq \sum_{j=1}^{m}\left(\beta_{j}^{+}-\beta_{j}^{-}\right) .
$$

Moreover, for every $j \in 1, \ldots, m$ there is a smooth curve $\gamma_{j}:[0,1] \rightarrow \Gamma_{k}^{j}$ such that

$$
|\dot{\gamma}|_{g}>0 \text { on }[0,1], \quad \operatorname{dist}\left(p_{k}, \gamma(0)\right)=\beta_{j}^{-} \quad \text { and } \quad \operatorname{dist}\left(p_{k}, \gamma(1)\right)=\beta_{j}^{+} .
$$

Consequently,

$$
\begin{aligned}
\beta_{j}^{+}-\beta_{j}^{-} & =\int_{0}^{1} \frac{d}{d s} \operatorname{dist}\left(p_{k}, \gamma(s)\right) d s=-\int_{0}^{1} \frac{1}{\left|\operatorname{Exp}_{\gamma(s)}^{-1}\left(p_{k}\right)\right|_{g}}\left\langle\operatorname{Exp}_{\gamma(s)}^{-1}\left(p_{k}\right), \dot{\gamma}(s)\right\rangle_{g} d s \\
& \leq \int_{0}^{1}|\dot{\gamma}(s)|_{g} d s \leq \sigma_{g}\left(\Gamma_{k}^{j}\right),
\end{aligned}
$$

the last inequality being a consequence of the fact that $\Gamma_{k}^{j}$ is a one-dimensional submanifold of $\mathcal{M}$. Here the assumption $N=2$ enters. Combining this estimate with (99), we deduce that

$$
\left|R_{k} \cap\left[2 r_{0}+\varepsilon, 3 r_{0}-\varepsilon\right]\right| \leq \sum_{j=1}^{m} \sigma_{g}\left(\Gamma_{k}^{j}\right) \leq \sigma_{g}\left(\Gamma_{k}\right)
$$

By considering the limit $\varepsilon \rightarrow 0$ we conclude that $\left|R_{k}\right| \leq \sigma_{g}\left(\Gamma_{k}\right)$, as claimed in (98). Combining this inequality with (96) gives (92). The proof is thus finished.

We may now complete the proof of Theorem 4.1 as follows (by contradiction):

By (88), Lemma 4.2(ii) and the variational characterization of $\nu_{2}\left(\Omega_{k}, g\right)$ we have that

$$
\limsup _{k \rightarrow \infty} \nu_{2}\left(\Omega_{k}, g\right) \leq \frac{\limsup _{k \rightarrow \infty} \int_{\Omega_{k}}\left|\nabla f_{k}\right|_{g}^{2} d v_{g}}{\liminf _{k \rightarrow \infty} \int_{\partial \Omega_{k}} f_{k}^{2} d \sigma_{g}} \leq \frac{c_{0}^{2} \lim _{k \rightarrow \infty}\left|\Omega_{k}\right|_{g}}{c_{1}}=0
$$

which contradicts (85). The proof of Theorem 4.1 is thus finished. 


\section{References}

[1] Brasco L.; De Philippis G.; Ruffini B., Spectral optimization for the Stekloff-Laplacian: the stability issue. J. Funct. Anal. 262 (2012), no. 11, 46754710.

[2] Brock F., An isoperimetric inequality for eigenvalues of the Stekloff problem, ZAMM Z. Angew. Math. Mech., 81 (2001), 69-71.

[3] Chavel I., Eigenvalues in Riemannian geometry, Academic Press, New York, 1984.

[4] Chavel I., Riemannian geometry. A modern introduction. Second edition. Cambridge Studies in Advanced Mathematics, 98. Cambridge University Press, Cambridge, 2006.

[5] Druet O., Asymptotic expansion of the Faber-Krahn profile of a compact Riemannian manifold. C. R. Math. Acad. Sci. Paris 346 (2008), no. 21-22, 1163-1167.

[6] Druet O., Sharp local isoperimetric inequalities involving the scalar curvature. Proc. Amer. Math. Soc. 130 (2002), no. 8, 2351-2361.

[7] Escobar, J. F., An isoperimetric inequality and the first Steklov eigenvalue. J. Funct. Anal. 165 (1999), 101-116.

[8] Fall M. M. and Weth T., Sharp local upper bound of the first non-zero Neumann eigenvalue in Riemannian manifolds. Calc. Var. Partial Differential Equations 51 (2014), no. 1-2, 217-242.

[9] Fall M. M. Some local eigenvalue estimates involving curvature. Calc. Var. Partial Differential Equations 36 (2009), no. 3, 437-451.

[10] Hadamard J., Mémoire sur le problème d'analyse relatif à l'équilibre des plaques elastiques encastrées, œuvres, tome 2, 1968.

[11] Jost, J., Riemannian geometry and geometric analysis. Sixth edition. Universitext. Springer, Heidelberg, 2011.

[12] Pacard F. and $\mathrm{Xu} \mathrm{X}$., Constant mean curvature spheres in Riemannian manifolds. Manuscripta Math. 128 (2009), no. 3, 275-295.

[13] Pacard F. and Sicbaldi P., Extremal domains for the first eigenvalue of the Laplace-Beltrami operator. Ann. Inst. Fourier (Grenoble) 59 (2009), no. 2, 515-542.

[14] Weinstock, R., Inequalities for a classical eigenvalue problem. J. Rational Mech. Anal. 3 (1954), 745-753.

[15] Xia, C. and Wang, Q., Inequalities for the Steklov eigenvalues. Chaos Solitons Fractals 48 (2013), 61-67. 